\title{
HUWE1 mediates inflammasome activation and promotes host defense against bacterial infection
}

\author{
Yu Guo, ${ }^{1,2}$ Longjun Li,,${ }^{1,3}$ Tao Xu, ${ }^{1}$ Xiaomin Guo, ${ }^{4}$ Chaoming Wang, ${ }^{1,3}$ Yihui Li, ${ }^{1,3}$ Yanan Yang, ${ }^{4}$ Dong Yang, ${ }^{5}$ Bin Sun, ${ }^{5}$ Xudong Zhao, \\ Genze Shao, ${ }^{6}$ and Xiaopeng $Q^{1}{ }^{1,4}$ \\ ${ }^{1}$ Key Laboratory of Animal Models and Human Disease Mechanisms of the Chinese Academy of Sciences/Key Laboratory of Bioactive Peptides of Yunnan Province, Kunming Institute of Zoology, Chinese \\ Academy of Sciences, Kunming, China. ${ }^{2}$ School of Life Sciences, University of Science and Technology of China, Hefei, China. ${ }^{3}$ Kunming College of Life Science, University of the Chinese Academy of Sciences, \\ Kunming, China. ${ }^{4}$ Advanced Medical Research Institute, Cheeloo College of Medicine, Shandong University, Jinan, China. ${ }^{5}$ Laboratory of Animal Tumor Models and Department of Thoracic Surgery, West China \\ Hospital, Sichuan University, Chengdu, China. ${ }^{6}$ Department of Cell Biology, School of Basic Medical Sciences, Peking University Health Science Center, Beijing, China.
}

\begin{abstract}
The mechanism by which inflammasome activation is modulated remains unclear. In this study, we identified an AIM2interacting protein, the E3 ubiquitin ligase HUWE1, which was also found to interact with NLRP3 and NLRC4 through the HIN domain of AIM2 and the NACHT domains of NLRP3 and NLRC4. The BH3 domain of HUWE1 was important for its interaction with NLRP3, AIM2, and NLRC4. Caspase-1 maturation, IL-1ß release, and pyroptosis were reduced in Huwe1-deficient bone marrow-derived macrophages (BMDMs) compared with WT BMDMs in response to stimuli to induce NLRP3, NLRC4, and AIM2 inflammasome activation. Furthermore, the activation of NLRP3, NLRC4, and AIM2 inflammasomes in both mouse and human cells was remarkably reduced by treatment with the HUWE1 inhibitor BI8622. HUWE1 mediated the K27-linked polyubiquitination of AIM2, NLRP3, and NLRC4, which led to inflammasome assembly, ASC speck formation, and sustained caspase-1 activation. Huwe1-deficient mice had an increased bacterial burden and decreased caspase-1 activation and IL-1ß production upon Salmonella, Francisella, or Acinetobacter baumannii infection. Our study provides insights into the mechanisms of inflammasome activation as well as a potential therapeutic target against bacterial infection.
\end{abstract}

\section{Introduction}

Inflammasomes are multiprotein complexes activated by infection and sterile stress that contribute to the initiation of pyroptotic cell death and release of the proinflammatory cytokines IL-1 $\beta$ and IL-18 $(1,2)$. Dysregulation of inflammasome activation causes a wide range of infectious and inflammatory diseases, cancer, and metabolic and autoimmune disorders $(3,4)$. Inflammasome activation and assembly are tightly regulated at multiple levels, and recent studies have significantly advanced our understanding of the regulation of inflammasome assembly and activation via the posttranslational modification of inflammasome components, such as ubiquitination and ubiquitin-like modifications (5-9). Ubiquitination is a highly complex and dynamic posttranslational protein modification that targets proteins for proteasomal degradation and interaction. Ubiquitination has multiple effects on the bacteria-host interface, and bacteria can also target host cell ubiquitination and ubiquitin-like modification pathways for invasion (10).

Initially, ubiquitination was reported to deliver inflammasome components to autophagosomes for destruction through recruitment of the autophagic adaptor p62 and ASC polyubiquitination (11). Increasing evidence indicates that both deubiquitina-

Authorship note: YG, LL, TX, and XG are co-first authors.

Conflict of interest: The authors have declared that no conflict of interest exists. Copyright: (5) 2020, American Society for Clinical Investigation.

Submitted: March 17, 2020; Accepted: August 11, 2020; Published: October 26, 2020

Reference information: J Clin Invest. 2020;130(12):6301-6316.

https://doi.org/10.1172/JCl138234. tion and ubiquitination can mediate inflammasome assembly and activation (12). NLRP3 deubiquitination mediated by the deubiquitinase BRCC3 was first demonstrated to be essential for inflammasome activation through the screening of a library of deubiquitinases and functional analysis (13). The deubiquitinases USP7 and USP47 were reported to regulate NLRP3 inflammasome activation by promoting ASC oligomerization and speck formation (14), and the deubiquitinase USP50 was reported to interact with ASC and remove the K63-linked ubiquitin of ASC to mediate ASC oligomerization and NLRP3 inflammasome activation (15). The E3 ligase MARCH7 was first reported to mediate the K48-linked polyubiquitination of NLRP3 and NLRP3 degradation, which contributed to the dopamine-mediated inhibition of NLRP3 inflammasome activation and neuroinflammation (16). NLRP3 inflammasome activation was also demonstrated to be negatively regulated by the E3 ligases TRIM31, cullin1, ARIH2, and FBXL2 (17-20) and positively modulated by TRAF3, TRAF6, and pellino2 (21-23). Regulation of the N-terminal domain of NLRP1B mediated by the Shigella flexneri E3 ubiquitin ligase IpaH7.8 and the E3 ligases UBR2 and UBR4 was found to be necessary and sufficient for NLRP1B activation (24-26).

Intracellular bacteria that have lost classical virulence factors, such as Francisella novicida, induce inflammasome activation mainly through engagement with the AIM2 receptor (27-29), but AIM2 inflammasome activation mediated by ubiquitination during bacterial infection has rarely been investigated (12). Inflammasome activation is a double-edged sword for infectious intracellular bacteria; it triggers an inflammatory response, but the repli- 
cation niche for intracellular bacteria is simultaneously reduced as a result of pyroptotic cell death. Therefore, inflammasome activation during intracellular bacterial infection needs to be tightly regulated at multiple levels. To define the mechanism by which the AIM2 inflammasome is modified during F. novicida infection, we performed mass spectrometry-based IP proteomics. Here, we identified an AIM2-interacting protein, the E3 ubiquitin ligase HUWE1, which was also found to interact with NLRP3 and NLRC4 but not with ASC or caspase-1. Huwe1-deficient primary bone marrow-derived macrophages (BMDMs) had impaired caspase- 1 activation in response to NLRP3, NLRC4, and AIM2 inflammasome activation stimuli. HUWE1 mediated the K27-linked polyubiquitination of AIM2, NLRP3, and NLRC4 and promoted ASC speck formation and the interaction between ASC and cleaved caspase-1. Huwe1-deficient mice had an increased bacterial burden and decreased caspase- 1 activation and IL- $1 \beta$ production under Salmonella, Francisella, or Acinetobacter baumannii infection. Our study reveals insights into the mechanisms of inflammasome activation, HUWE1-mediated cell death, and DNA damage responses and provides a potential therapeutic target against bacterial infection, inflammatory diseases, and even tumorigenesis.

\section{Results}

HUWE1 interacts with AIM2, NLRP3, and NLRC4. To investigate the regulation of AIM2 inflammasome activation upon $F$. novici$d a$ infection, we performed AIM2 IP-mass spectrometry (IP-MS) analysis of F. novicida-infected WT and Aim $2^{--}$BMDMs. By analyzing the protein composition of IP products from WT and Aim2 $2^{-/}$BMDMs, we identified an E3 ligase, HUWE1, present in the IP products of WT, but not Aim2 ${ }^{--}$, BMDMs (Supplemental Figure 1, A and B, and Supplemental Table 1; supplemental material available online with this article; https://oi.org/10.1172/ JCI138234DS1), suggesting that HUWE1 is a regulator of AIM2 inflammasome activation. To confirm the interaction between HUWE1 and AIM2, we performed co-IP analysis of HEK293T cells. Notably, we found that AIM2 indeed interacted with HUWE1 in transfected HEK293T cells (Figure 1A). Furthermore, HUWE1 also interacted with NLRP3 and NLRC4, the receptors of the other 2 important inflammasome complexes (Figure 1, A and B). However, HUWE1 did not interact with ASC or caspase-1, which are central components of the inflammasome complex (Supplemental Figure 1, C and D), indicating that HUWE1 does not directly interact with the PYD or CARD domains (Figure 1C). To determine whether the HIN, NACHT, or LRR domains mediate the interaction between HUWE1 and AIM2, NLRP3, or NLRC4, we performed co-IP analysis of HUWE1 and proteins expressing specific domains (Figure 1C). Interestingly, we found that the HIN domain of AIM2 and the NACHT domains of NLRP3 and NLRC4 were essential for the interactions between HUWE1 and AIM2, NLRP3, and NLRC4 (Figure 1D). However, neither the LRR domain of NLRP3 nor the that of NLRC4 interacted with HUWE1 (Supplemental Figure 1E). To further define the domain of HUWE1 that interacts with NLRP3, AIM2, and NLRC4, we performed co-IP analyses with truncated HUWE1 and NLRP3, AIM2, and NLRC4 (Figure 1E). We found the $\mathrm{BH} 3$ domain-containing fragment (F4, aa 1695-2028) to be critical for the interaction of HUWE1 with NLRP3, AIM2, and NLRC4 (Figure 1, F-H, and Supplemental Fig- ure 2, A-D). In addition, the interactions between F4 and the HIN domain of AIM2, and F4 and the NACHT domains of NLRP3 and NLRC4 were confirmed by co-IP analysis (Supplemental Figure 2E). Taken together, these data indicate that HUWE1 interacts with AIM2, NLRP3, and NLRC4 through the HIN and NACHT domains of inflammasome receptors.

HUWE1 mediates NLRP3, AIM2, and NLRC4 inflammasome activation. To determine whether HUWE1 plays a role in activating the NLRP3, AIM2, and NLRC4 inflammasomes, we conducted inflammasome activation analysis in WT and Huwe1 gene-deleted BMDMs. Given that the Huwe1 gene is located on the X chromosome in the mouse genome, we used BMDMs from mice of the same sex for all comparisons. Treatment of Huwe ${ }^{f / Y}$-CreER BMDMs with 4-hydroxytamoxifen (4-OHT) for 5 days induced deletion of the Huwe1 gene (Supplemental Figure 3, A and B). Then, Huwe $1^{+/ Y}$ and Huwe ${ }^{f / Y}$ BMDMs after 4-OHT treatment were stimulated with LPS and ATP for NLRP3 inflammasome activation; transfected with dsDNA; or infected with $F$. novicida for AIM2 inflammasome activation, infected with Salmonella for NLRC4 inflammasome activation, or infected with Listeria for both NLRP3 and AIM2 inflammasome activation (30-32). Remarkably, we noted a substantial reduction of caspase-1 activation of NLRP3, AIM2, and NLRC4 inflammasomes in Huwe ${ }^{f / Y}$ BMDMs compared with $H u w e 1^{+/ Y}$ BMDMs (Figure 2A). Consistent with the caspase-1 activation data, the production of IL-1 $\beta$ and cell death were significantly reduced in the absence of HUWE1 (Figure 2, B and C, and Supplemental Figure 3C). However, the production of TNF and IL-6 was not affected by HUWE1 (Supplemental Figure 3D). In addition, the expression levels of Illa and Il1b induced by LPS and poly(I:C) treatment and $F$. novicida infection were comparable between $H u w e 1^{+/ Y}$ and Huwe $1^{f / Y}$ BMDMs (Supplemental Figure 3E), indicating that HUWE1 directly regulates inflammasome activation and inflammatory responses independently of NF- $\kappa \mathrm{B}$ signaling. To confirm this observation, we used monocyte/macrophage-specific deletion of the Huwe1 gene in Lyz2-Cre mice (Supplemental Figure $3 \mathrm{~F}$ ). Consistently, caspase- 1 activation, IL-1 $\beta$ production, and cell death due to NLRP3, AIM2, and NLRC4 inflammasome activation were remarkably reduced in Lyz2-Cre-expressing Huwe $1^{f / Y}$ BMDMs compared with Lyz2-Cre-expressing Huwe $1^{+/ Y}$ BMDMs (Figure 2, D-F). Instead, the production of TNF and IL-6 was comparable between Lyz2-Cre-expressing Huwe $1^{f / Y}$ BMDMs and Lyz2Cre-expressing Huwe ${ }^{+/ Y}$ BMDMs (Supplemental Figure 3G).

To further examine the roles of HUWE1 in inflammasome activation, we treated cells with the HUWE1-specific inhibitor BI8622 (33). Interestingly, caspase-1 activation in NLRP3, AIM2, and NLRC4 inflammasomes was substantially reduced in BMDMs treated with BI8622 compared with untreated BMDMs (Figure $3 A)$. IL-1 $\beta$ production and cell death in response to treatment with LPS and ATP or infection with F. novicida, Salmonella, or Listeria were significantly reduced in BMDMs treated with BI8622 (Figure $3, \mathrm{~B}$ and $\mathrm{C})$. The level of IL- $1 \beta$ in BMDMs transfected with dsDNA was very low owing to the short treatment duration (Figure 3B). In addition, we found that IL- $1 \beta$ production and cell death were significantly reduced in human THP-1 cells and PBMCs in the presence of BI8622 treatment (Figure 3, D-G). These results collectively indicate that HUWE1 mediates NLRP3, AIM2, and NLRC4 inflammasome activation. 
A
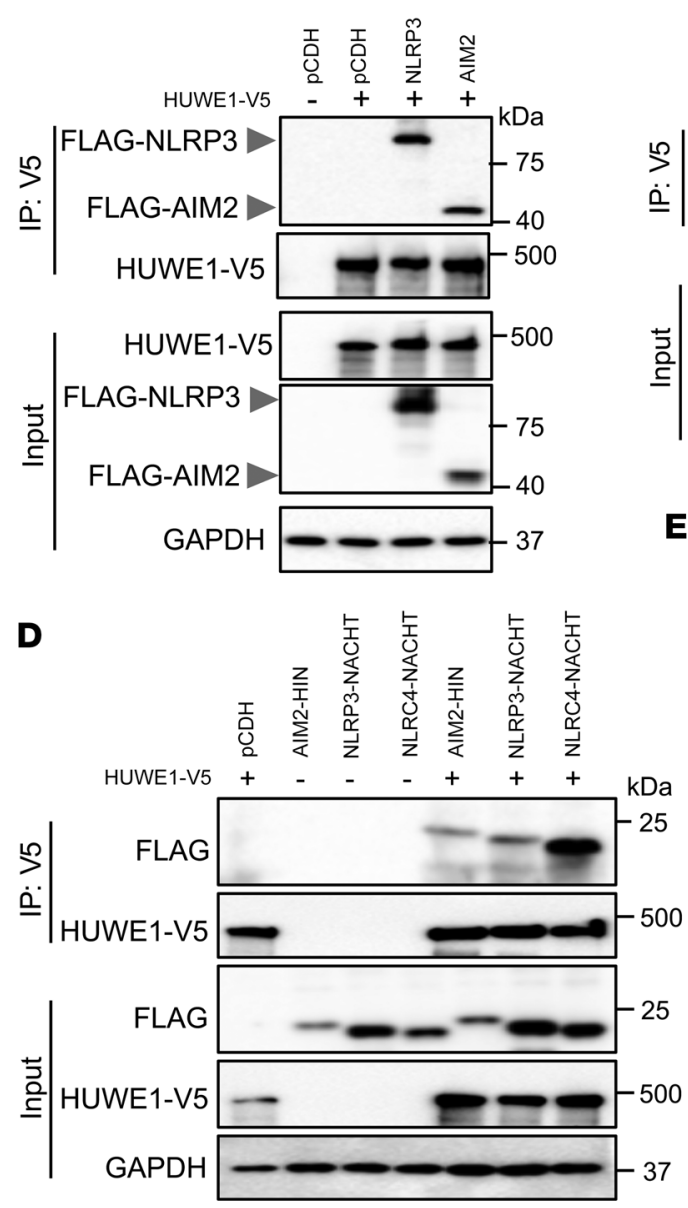

G

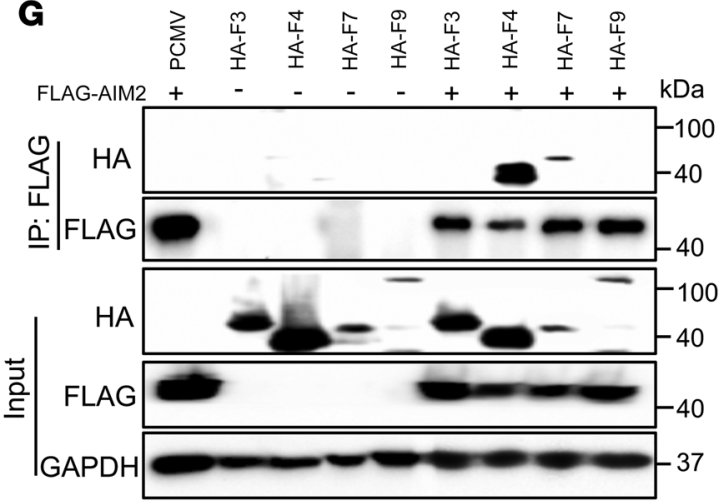

B

NLRC4

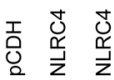

HUWE1-V5 $+\quad+$

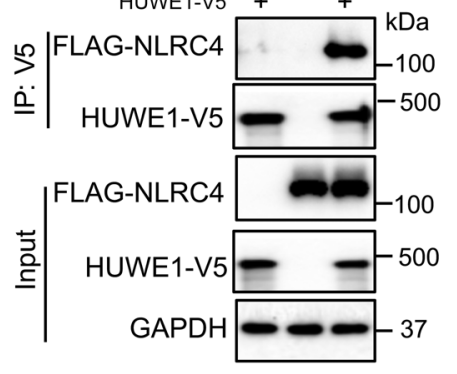

E ARLD1 ARLD2 UBA WWE BH3 NLS F7 (2368-2654) F9 $\underline{\text { (3551-4374) }}$

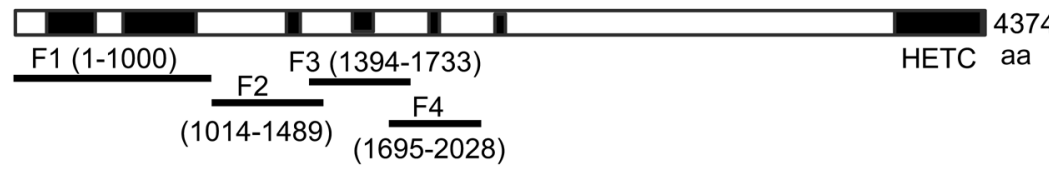

\section{AIM2 PYD-HIN}

NLRP3 PYD-NACHT LRR

NLRC4 CARD-NACHT LRR

ASC PYD CARD

Caspase-1 CARD-CASP
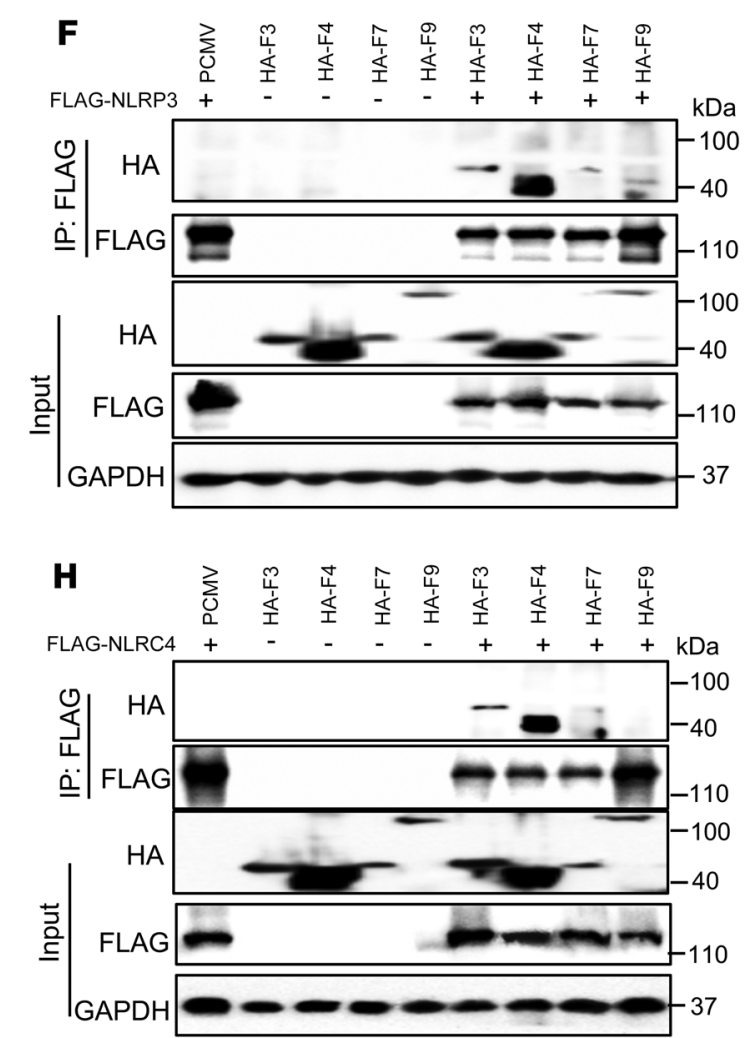

Figure 1. HUWE1 interacts with NLRP3, AIM2, and NLRC4. (A and B) Immunoblot analysis of HUWE1 co-IP with FLAG-NLRP3, FLAG-AIM2 (A), and FLAG-NLRC4 (B) from lysates of HEK293T cells transfected with the indicated plasmids. (C) Illustration of AIM2, NLRP3, NLRC4, ASC, and caspase-1 domains. (D) Immunoblot analysis of HUWE1 co-IP with FLAG-AIM2-HIN, FLAG-NLRP3-NACHT, and FLAG-NLRC4-NACHT from lysates of HEK293T cells transfected with the indicated plasmids. (E) Schematic representation of fragments from HUWE1 protein. (F-H) Immunoblot analysis of FLAG-NLRP3 (F), FLAG-AIM2 (G), and FLAG-NLRC4 (H) interaction with HA-tag-fused HUWE1 fragments (F3, F4, F7, and F9) from lysates of HEK293T cells transfected with the indicated plasmids. Data are representative of 3 independent experiments.

HUWE1 regulates the K27-linked polyubiquitination of AIM2, NLRP3, and NLRC4. To determine whether HUWE1 mediates the ubiquitination of NLRP3, AIM2, and NLRC4, we transfected HEK293T cells with HUWE1; NLRP3, AIM2, or NLRC4; and
HA-tagged WT ubiquitin or K6-, K11-, K27-, K29-, K33-, K48-, or K63-specific ubiquitin. Remarkably, HUWE1 selectively promoted the WT and K27-linked polyubiquitination of NLRP3 and AIM2 and preferentially mediated the K27-linked polyubiquitination of NLRC4 


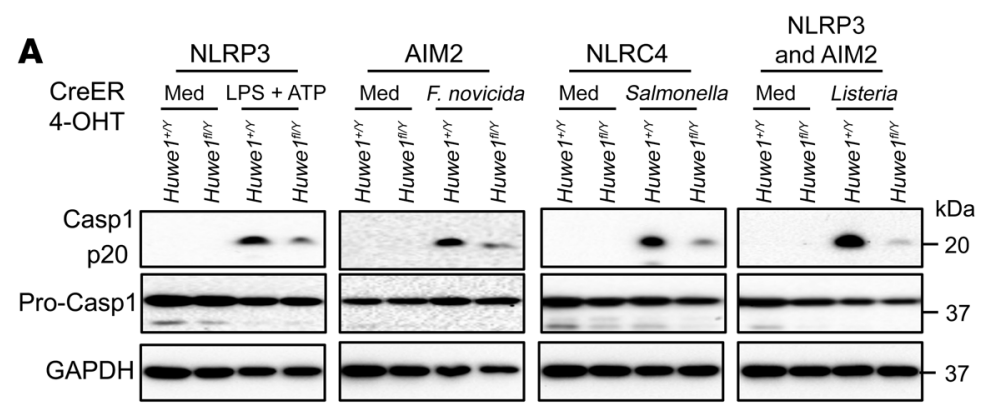

B

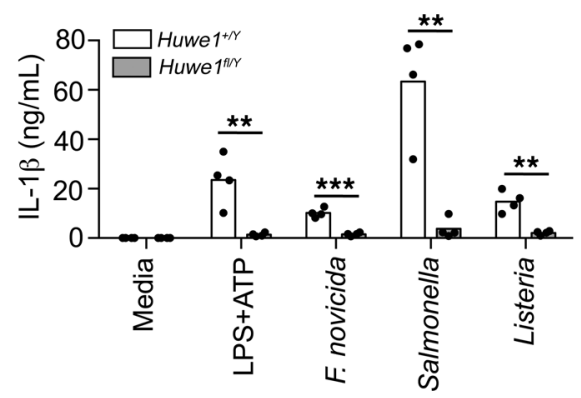

c
CreER 4-OHT

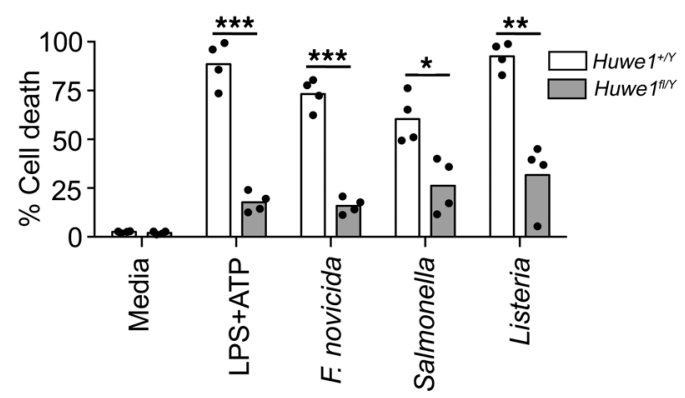

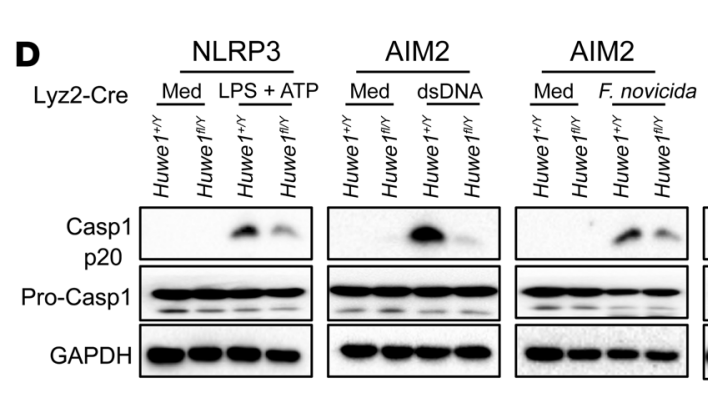

NLRP3
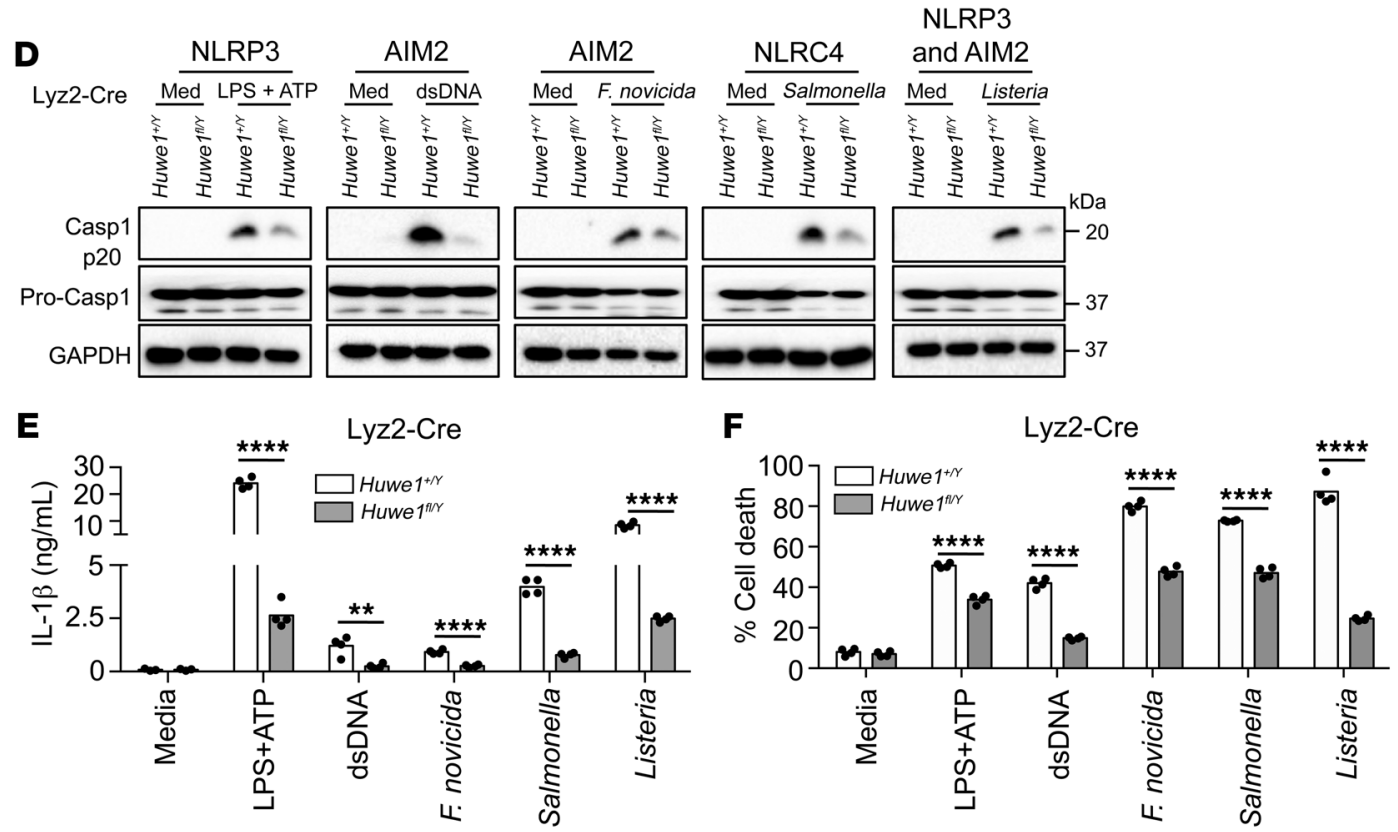

Figure 2. HUWE1 deficiency impairs NLRP3, AIM2, and NLRC4 inflammasome activation. (A-C) CreER-expressing Huwe $7^{+/ Y}$ and Huwe ${ }^{f / / \gamma}$ BMDMs were treated with 4-OHT (100 nM) for 5 days to induce Huwe1 gene deletion. Immunoblot analysis of pro-caspase-1 (Pro-Casp1) and its subunit p20 (A) and

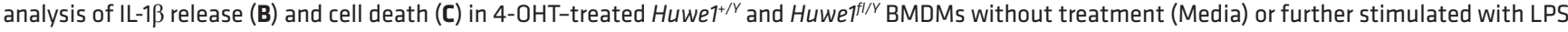
( $500 \mathrm{ng} / \mathrm{mL}, 4 \mathrm{~h}$ ) and ATP (5 mM, $50 \mathrm{~min}$ ) for NLRP3 inflammasome activation, or infected with F. novicida (200 MOI, 16 h) for AIM2 inflammasome activation, or Salmonella (3 MOI, 2 h) for NLRC4 inflammasome activation, or Listeria (50 MOI, 3 h) for both NLRP3 and AIM2 inflammasome activation. (D-F) Immunoblot analysis of pro-caspase-1 (Pro-Casp1) and its subunit p20 (D) and analysis of IL-1 $\beta$ release (E) and cell death (F) in Lyz2-Cre-expressing

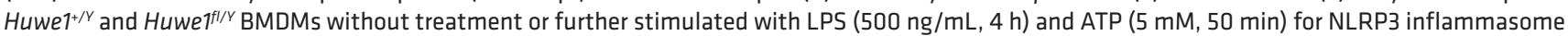
activation; or transfected with dsDNA $(0.5 \mu \mathrm{g}, 8 \mathrm{~h})$ for AIM2 inflammasome activation; or infected with F. novicida (200 MOI, $16 \mathrm{~h}$ ) for AIM2 inflammasome activation, Salmonella (3 MOI, 2 h) for NLRC4 inflammasome activation, or Listeria ( $50 \mathrm{MOI}, 3 \mathrm{~h}$ ) for both NLRP3 and AIM2 inflammasome activation. Each dot represents an individual experiment (B, C, E, and $\mathbf{F})$. ${ }^{*} P<0.05$, ${ }^{* *} P<0.01$, ${ }^{* * *} P<0.001$, and ${ }^{* * * *} P<0.0001$, by 2 -sided Student's $t$ test without multiple-comparisons correction. Data are representative of 3 (A and $\mathbf{D})$ or 4 (B, C, E, and $\mathbf{F}$ ) independent experiments. Med, media.

(Figure 4, A-C, and Supplemental Figure 4, A-C). Furthermore, HUWE1 did not catalyze the polyubiquitination of NLRP3, AIM2, or NLRC4 in the presence of K27R-mutant ubiquitin, which harbored a lysine-to-arginine substitution at position 27 (Figure 4, A-C).
To determine which lysine residues of NLRP3, AIM2, and NLRC4 are essential for their HUWE1-mediated K27-linked polyubiquitination, we investigated the ubiquitination of the HIN domain of AIM2 and the NACHT domains of NLRP3 and NLRC4, 
A
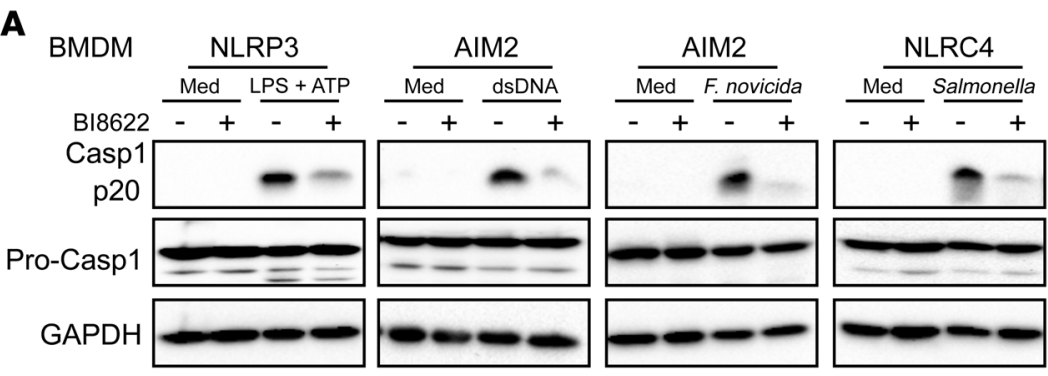

NLRP3

and AIM2

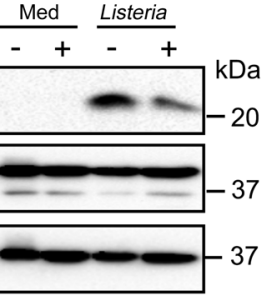

B

BMDM

D

THP-1

$\mathbf{F}$

Human PBMC
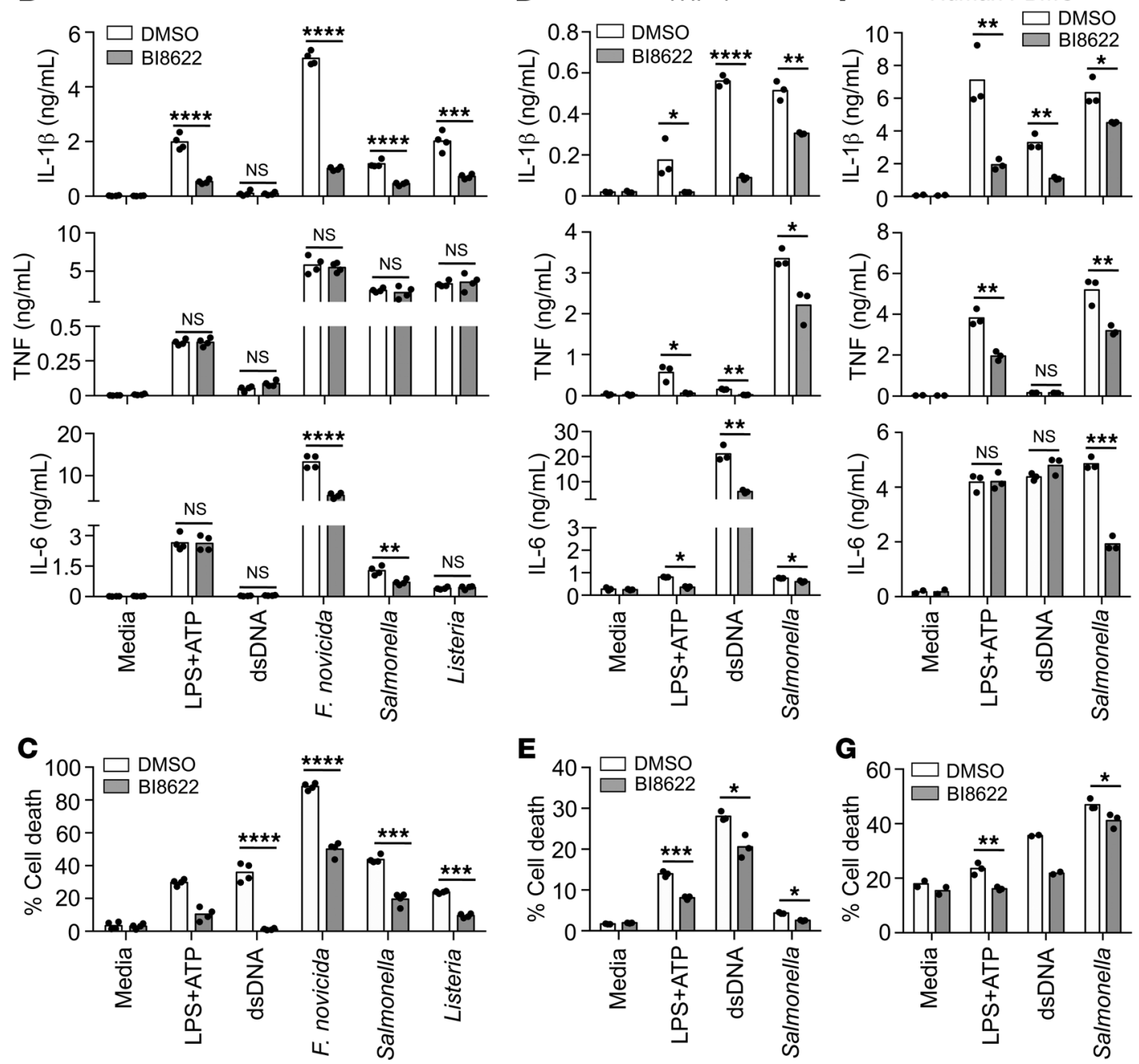

Figure 3. The HUWE1 inhibitor B18622 reduces NLRP3, AIM2, and NLRC4 inflammasome activation. (A-C) Immunoblot analysis of pro-caspase-1 and its subunit p20 (A), analysis of IL-1 $\beta$ release and TNF and IL-6 production (B), and analysis of cell death (C) in control DMSO-treated or BI8622-treated WT BMDMs without treatment or further stimulated with LPS ( $500 \mathrm{ng} / \mathrm{mL}, 4 \mathrm{~h})$ and ATP $(5 \mathrm{mM}, 50 \mathrm{~min})$ for NLRP3 inflammasome activation; or transfected with dsDNA $(1.5 \mu \mathrm{g}, 2 \mathrm{~h}$ ) for AIM2 inflammasome activation; or infected with F. novicida (200 MOI, $16 \mathrm{~h}$ ) for AIM2 inflammasome activation, Salmonella (3 MOI, 2 h) for NLRC4 inflammasome activation, or Listeria (50 MOI, 3 h) for both NLRP3 and AIM2 inflammasome activation. (D and E) Analysis of IL-1 3 release and TNF and IL-6 production (D) and cell death $(\mathbf{E})$ in control DMSO-treated or BI8622-treated THP-1 cells without treatment or further stimulated with LPS $(1 \mu \mathrm{g} / \mathrm{mL}, 6 \mathrm{~h})$ and ATP ( $10 \mathrm{mM}, 2 \mathrm{~h})$; or transfected with dsDNA $(1.5 \mu \mathrm{g}, 10 \mathrm{~h})$; or infected with Salmonella $(6 \mathrm{MOI}, 6 \mathrm{~h})$. (F and G) Analysis of IL-1 $\beta$ release and TNF and IL-6 production (F) and cell death (G) in control DMSO-treated or BI8622-treated human PBMCs without treatment or further stimulated with LPS $(1 \mu \mathrm{g} / \mathrm{mL}, 6 \mathrm{~h})$ and ATP $(10 \mathrm{mM}, 2 \mathrm{~h})$; or transfected with dsDNA (1.5 $\mu \mathrm{g}, 10 \mathrm{~h})$; or infected with Salmonella (6 MOI, $6 \mathrm{~h})$. Each dot represents an individual experiment. ${ }^{*} P<0.05$, ${ }^{* *} P<0.01$, ${ }^{* *} P<0.001$, and ${ }^{* * * *} P<0.0001$, by 2 -sided Student's $t$ test without multiple-comparisons correction. Data are representative of $3(\mathbf{A}$ and $\mathbf{D}-\mathbf{C})$ or 4 ( $\mathbf{B}$ and $\mathbf{C}$ ) independent experiments. 

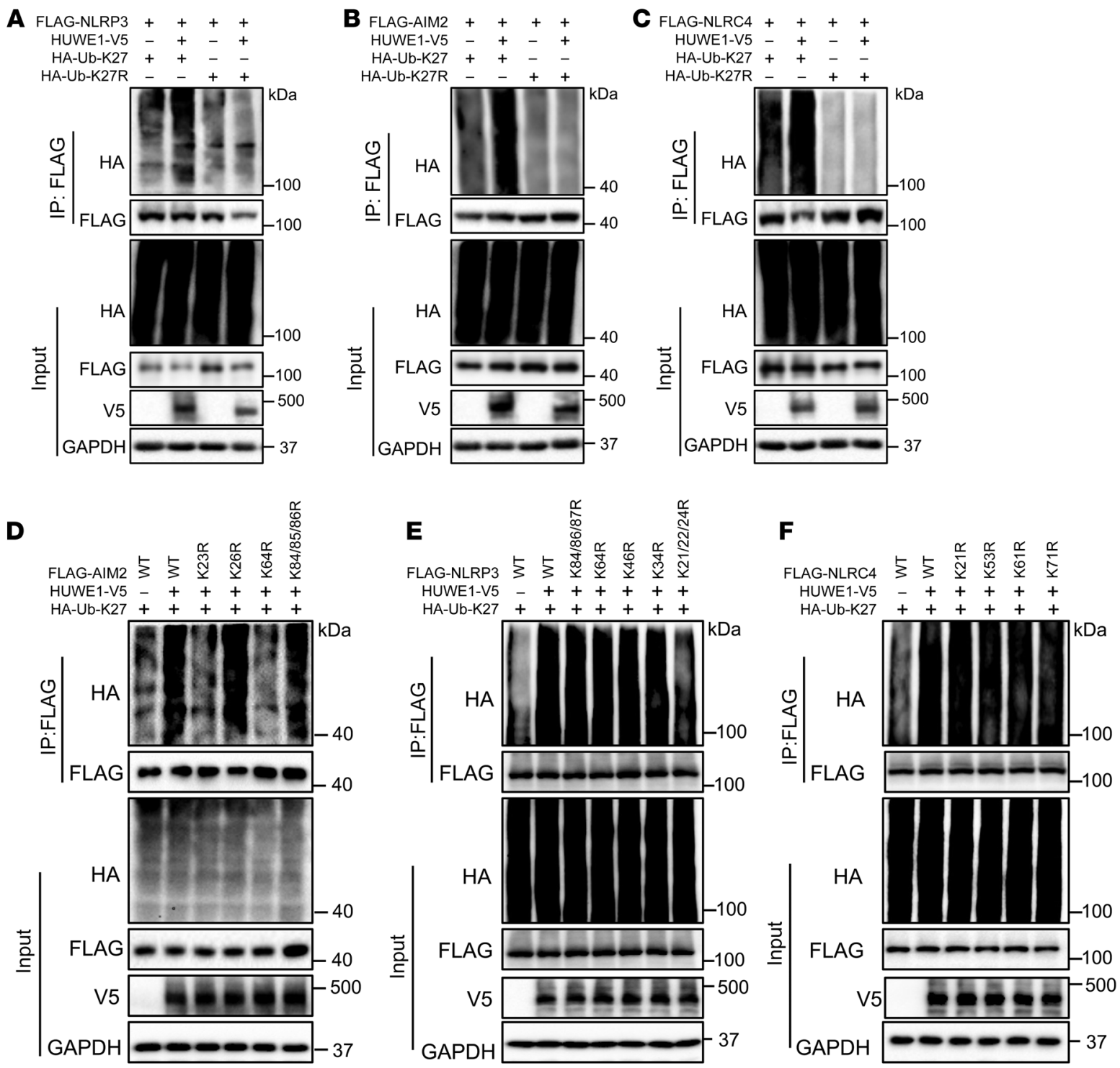

Figure 4. HUWE1 mediates the K27-linked polyubiquitination of NLRP3, AIM2, and NLRC4. (A-C) Co-IP analysis of WT K27- and K27-mutant (K27Rlinked) polyubiquitination of NLRP3 (A), AIM2 (B), and NLRC4 (C) mediated by HUWE1 in 293T cells transfected with the indicated plasmids. (D-F) Co-IP analysis of the polyubiquitination of WT AIM2 and AIM2 mutants (K23R, K26R, K64R, and K84R/K85R/K86R) (D), WT NLRP3 and NLRP3 mutants (K84R/K86R/K87R, K64R, K46R, K34R, and K21R/K22R/K24R) (E), and WT NLRC4 and NLRC4 mutants (K21R, K53R, K61R, and K71R) (F) mediated by HUWE1 in 293T cells transfected with the indicated plasmids. Data are representative of 3 independent experiments. Ub, ubiquitin.

which were found to directly interact with HUWE1 (Figure 1D). However, HUWE1 did not mediate K27-linked polyubiquitination of the HIN or NACHT domains (Supplemental Figure 4D). These data suggested that the lysine residues within the PYD domain of AIM2 are critical for HUWE1-mediated AIM2 ubiquitination. To pinpoint the residues required for AIM2 ubiquitination, we generated 4 AIM2 proteins with site-directed mutations in the PYD domain (K23R, K26R, K64R, K84R/K85R/K86R). Notably, K27linked polyubiquitination of the K23R and K64R AIM2 mutants was diminished, but that was not the case in the other AIM2 mutants (Figure 4D). Similarly, we also generated NLRP3 proteins with site-directed mutations in the N-terminal PYD domain and NLRC4 proteins with site-directed mutations in the N-terminal CARD domain and found that the K21, K22, and K24 sites of
NLRP3 and the K61 and K71 sites of NLRC4 were important for HUWE1-mediated K27-linked polyubiquitination of NLRP3 and NLRC4, respectively (Figure 4, E and F).

HUWE1 promotes NLRP3, AIM2, and NLRC4 inflammasome activation in HEK293T cells. In HEK293T cells, the NLRP3 inflammasome can be reconstituted by the ectopic expression of procaspase-1, NLRP3, and ASC with the appropriate stimulation (34). To determine whether HUWE1 can promote NLRP3 inflammasome activation in HEK293T cells, we transfected HEK293T cells with pro-caspase-1, NLRP3, and ASC with or without HUWE1 and stimulated the cells with nigericin. Interestingly, the activation of caspase-1, as indicated by blotting for $\mathrm{p} 20$, was significantly increased in HUWE1-transfected cells compared with vector control-transfected cells (Figure 5A). HEK293T cells were transfected 

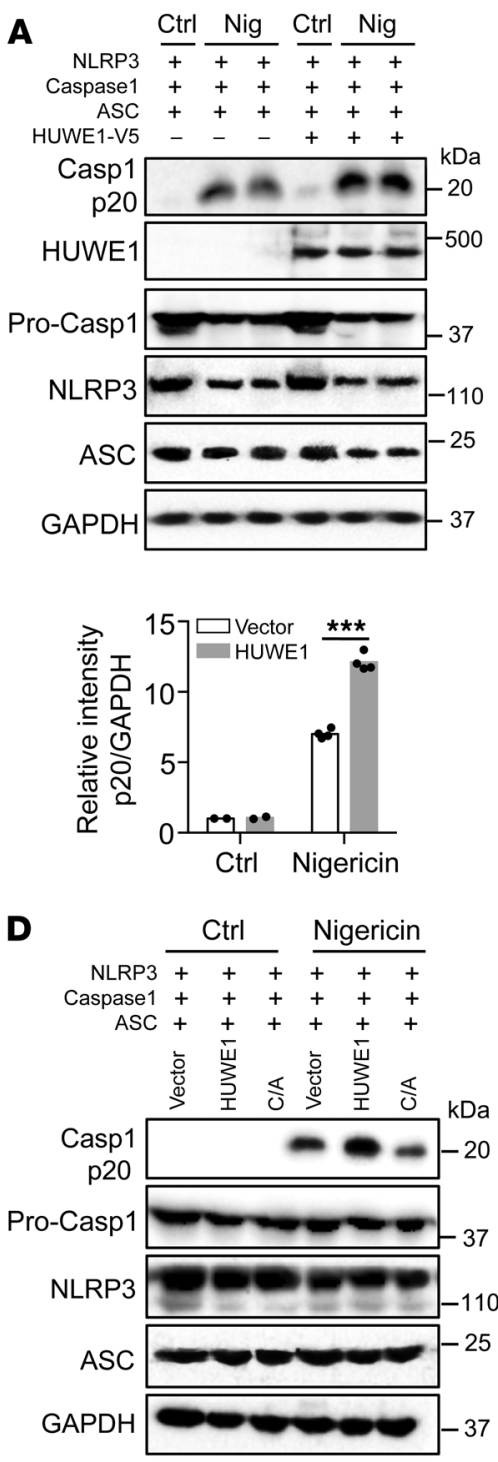

B
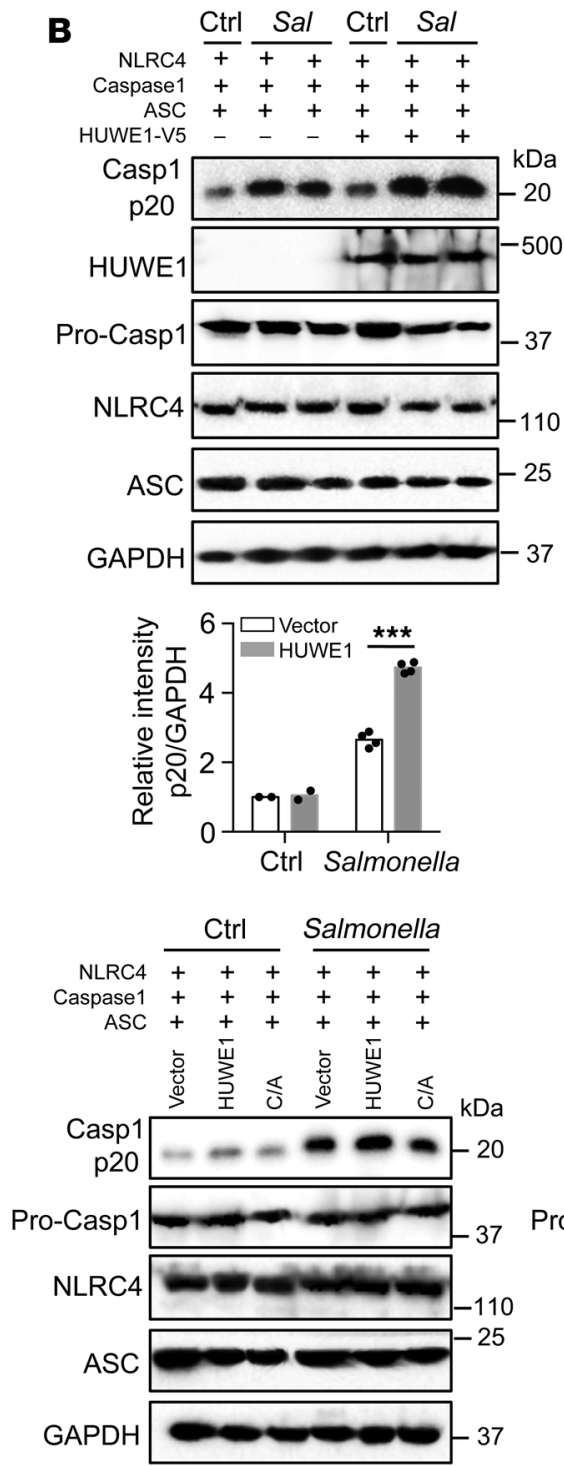

C
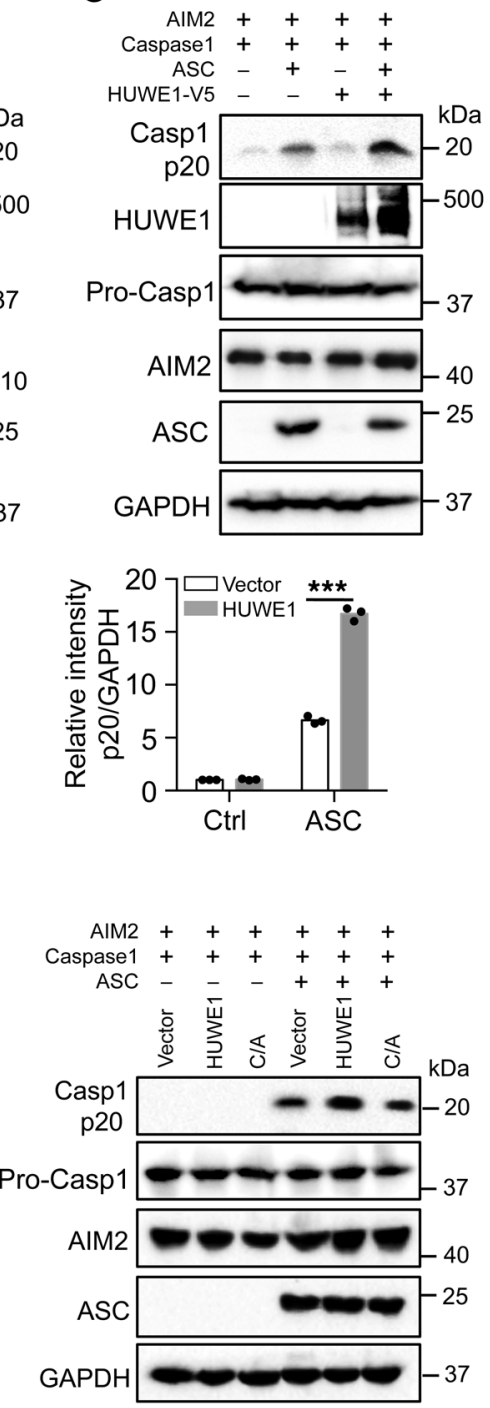

Figure 5. HUWE1 increases NLRP3, AIM2, and NLRC4 inflammasome activation in 293T cells. (A) Immunoblot analysis of pro-caspase-1 and its subunit p20, HUWE1, NLRP3, and ASC in 293T cells transfected with the indicated plasmids followed by control (Ctrl) or nigericin (Nig) stimulation (10 $\mu \mathrm{g} / \mathrm{mL})$ for 2 hours. Plot shows quantification analysis of the signaling intensity of p20 relative to GAPDH. (B) Immunoblot analysis of pro-caspase-1 and its subunit p20, HUWE1, NLRC4, and ASC in 293T cells transfected with the indicated plasmids followed by Salmonella (Sal) infection (3 MOI) for 2 hours. Plot shows quantification analysis of the signaling intensity of p20 relative to GAPDH. (C) Immunoblot analysis of pro-caspase- 1 and its subunit p20, HUWE1, AIM2, and ASC in 293T cells stably expressing AIM2 and caspase-1, transfected with HUWE1 and ASC as indicated. Plot shows quantification analysis of the signaling intensity of p20 relative to GAPDH. (D) Immunoblot analysis of pro-caspase-1 and its subunit p20 in $293 T$ cells transfected with WT HUWE1 or the HUWE1 C4341A mutant (C/A) and other plasmids as indicated, followed by stimulation as in A-C. Each dot represents an individual experiment (A-C). ${ }^{* * *} P<0.001$, by 2 -sided Student's $t$ test without multiple-comparisons correction. Data are representative of 3 independent experiments.

with pro-caspase-1, NLRC4, and ASC with or without HUWE1 and infected with Salmonella, which showed that HUWE1 also significantly increased NLRC4 inflammasome activation (Figure 5B). AIM2 is an intracellular DNA sensor (35-38) that triggers AIM2 inflammasome activation in response to DNA transfection. To avoid the effect of DNA transfection on AIM2 inflammasome activation, we established HEK293T cells that stably expressed AIM2 and pro-caspase- 1 by lentiviral transfection and further transfected the cells with HUWE1 for 24 hours. Finally, ASC was transfected into these cells, and the activation of caspase- 1 was analyzed. Consistent with the NLRP3 and NLRC4 data, we found that HUWE1 indeed increased AIM2 inflammasome activation (Figure 5C). The
HUWE1 catalytic site mutant C4341A (C/A) failed to promote the activation of NLRP3, AIM2, and NLRC4 inflammasomes (Figure 5D), indicating that the ubiquitination mediated by HUWE1 was essential for the inflammasome activation.

To define the role of HUWE1-mediated ubiquitination activity in NLRP3, AIM2, and NLRC4 inflammasome activation, HEK293T cells were transfected with WT HUWE1, pro-caspase-1, or ASC and their mutant receptors, followed by stimulation. Remarkably, the NLRP3 ubiquitination site mutant K21R/K22R/K24R reduced caspase-1 activation (Figure 6A). Activation of the AIM2 and NLRC4 inflammasomes was also substantially inhibited in the presence of the K23R and K64R AIM2 mutants and the K61R and K71R NLRC4 
A
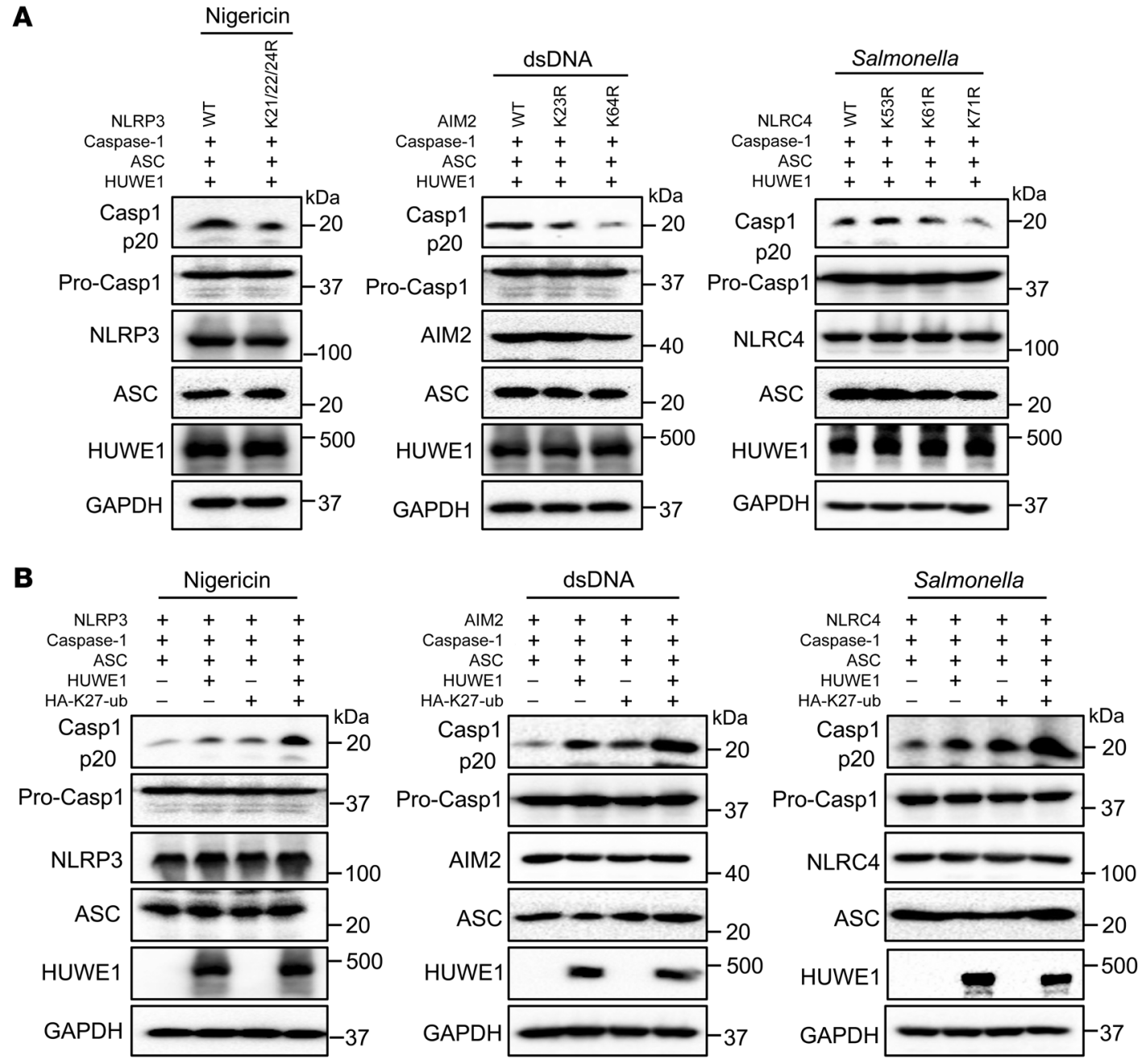

Figure 6. K27 ubiquitin promotes HUWE1-mediated NLRP3, AIM2, and NLRC4 inflammasome activation. (A) Immunoblot analysis of pro-caspase-1 and its subunit p20 in 293T cells transfected with WT NLRP3, AIM2, NLRC4 and their mutants in the presence of HUWE1 and other plasmids as indicated, followed by stimulation for NLRP3 (nigericin, $10 \mu \mathrm{g} / \mathrm{mL}, 2$ hours), AIM2 (dsDNA, $2 \mu \mathrm{g} / \mathrm{mL}, 2$ hours), and NLRC4 (Salmonella, 3 MOI, 2 hours) inflammasome activation. (B) Immunoblot analysis of pro-caspase-1 and its subunit p20 in 293T cells transfected with WT NLRP3, AIM2, and NLRC4 in the presence or absence of HUWE1 and K27 ubiquitin and other plasmids as indicated, followed by stimulation for NLRP3, AIM2, and NLRC4 inflammasome activation as in $\mathbf{A}$. Data are representative of 3 independent experiments.

mutants, respectively (Figure 6A). To further confirm the role of K27 ubiquitin in HUWE1-mediated inflammasome activation, we transfected HEK293T cells with HUWE1, pro-caspase-1, ASC, and receptors in the presence or absence of $\mathrm{K} 27$ ubiquitin, followed by stimulation. Interestingly, K27 ubiquitin dramatically increased HUWE1-mediated activation of NLRP3, AIM2, and NLRC4 inflammasomes (Figure 6B). These data collectively indicate that HUWE1 promotes NLRP3, AIM2, and NLRC4 inflammasome activation through mediation of K27-linked polyubiquitination.

HUWE1 regulates the endogenous ubiquitination of NLRP3, AIM2, and NLRC4 and ASC speck formation. To confirm the interaction between endogenous HUWE1 and inflammasome receptors, we performed co-IP analysis of HUWE1 and NLRP3, AIM2, and NLRC4 in Lyz2-Cre-expressing Huwe $1^{+/ Y}$ and Lyz2Cre-expressing Huwe $1^{f / Y}$ BMDMs with and without stimulation. Strikingly, we observed that NLRP3, AIM2, and NLRC4 were immunoprecipitated by HUWE1 in Lyz2-Cre-expressing Huwe $1^{+/ Y}$
BMDMs, but not Lyz2-Cre-expressing Huwe $1^{f / Y}$ BMDMs, under the condition of inflammasome activation (Figure 7A). The expression of NLRP3, AIM2, and NLRC4 was induced after stimulation, which might have contributed to their interaction with HUWE1 in the stimulated conditions (Figure 7A). To determine whether the ubiquitination of endogenous NLRP3, AIM2, and NLRC4 is mediated by HUWE1, we performed endogenous IP and ubiquitination analysis of Lyz2-Cre-expressing Huwe ${ }^{+/ Y}$ and Lyz2-Cre-expressing Huwe $1^{f / Y}$ BMDMs after stimulation. Indeed, the ubiquitination of endogenous NLRP3, AIM2, and NLRC4 was substantially reduced in the absence of HUWE1 (Figure 7B).

To identify the mechanism by which the ubiquitination of these receptors contributes to inflammasome activation, we examined the oligomerization of NLRP3 in BMDMs by disuccinimidyl suberate (DSS) cross-linking analysis. The results revealed that HUWE1 mediated the oligomerization of NLRP3 in BMDMs (Figure 8A). Interestingly, HUWE1 and NLRP3 were colocalized 
A

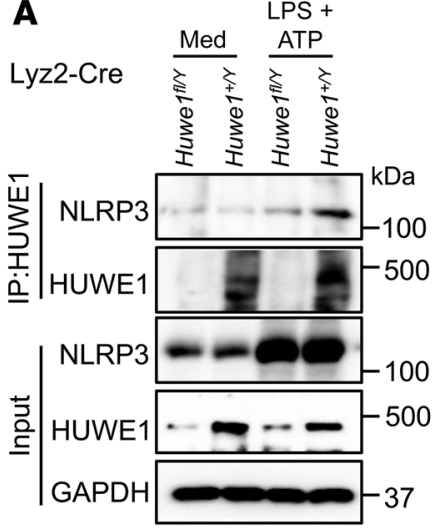

B

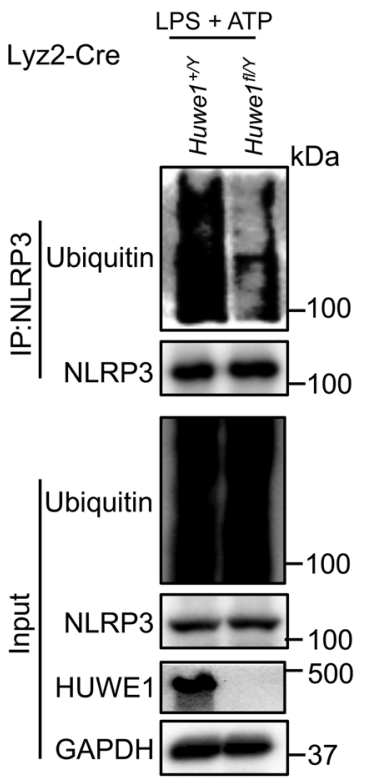

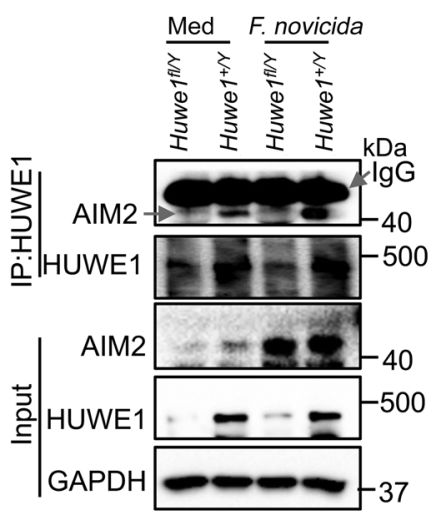
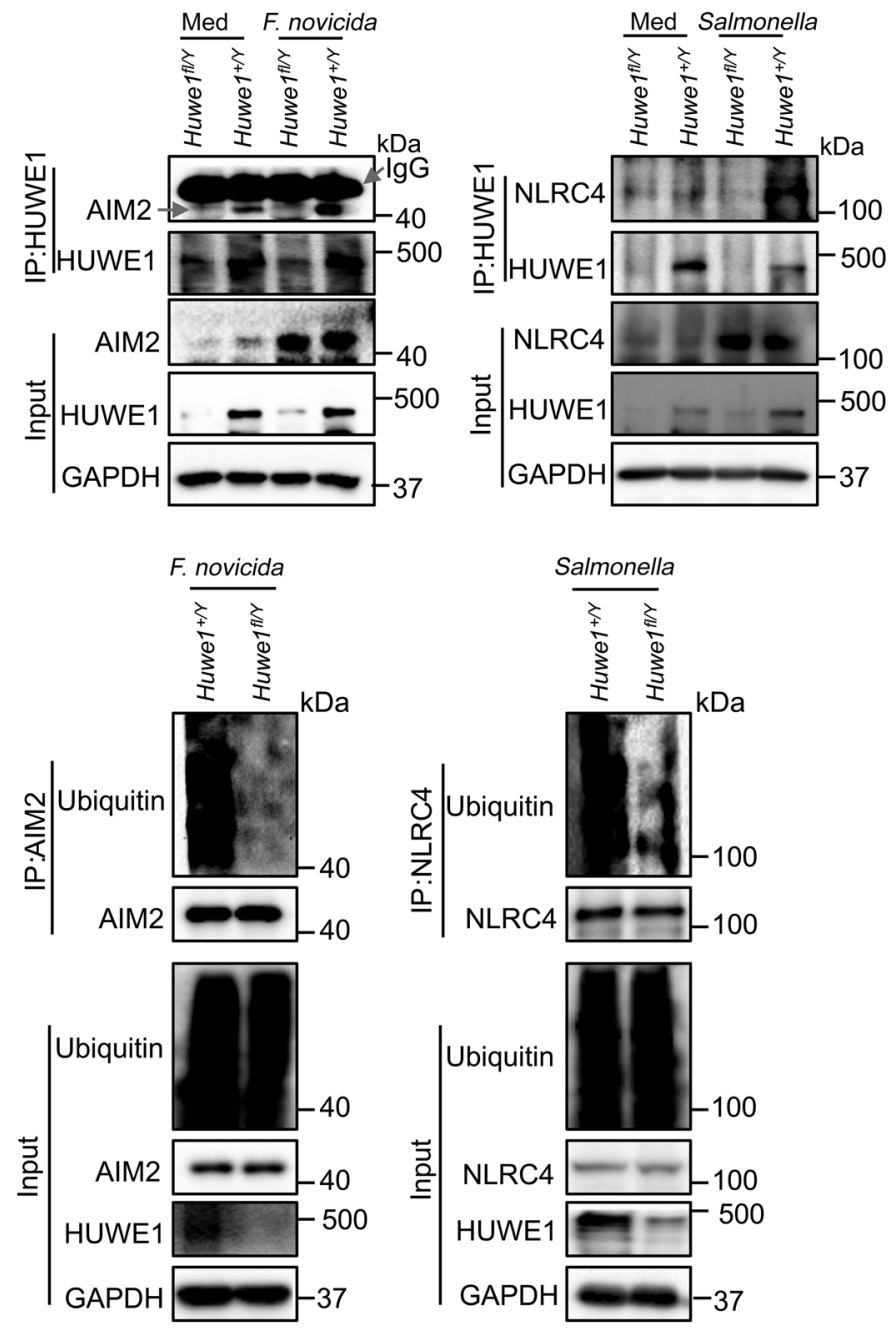

Figure 7. HUWE1 interacts with endogenous NLRP3, AIM2, and NLRC4 and regulates their ubiquitination. (A) Co-IP analysis of endogenous HUWE1 and NLRP3, AIM2, and NLRC4 in Lyz2-Creexpressing Huwe ${ }^{+/ / V}$ and Huwe $7^{f / Y}$ BMDMs with or without NLRP3 inflammasome stimulation (LPS, $500 \mathrm{ng} / \mathrm{mL}, 4 \mathrm{~h}$ and ATP, $5 \mathrm{mM}, 10$ min), AIM2 inflammasome stimulation (F. novicida, $100 \mathrm{MOI}, 12 \mathrm{~h}$ ), or NLRC4 inflammasome stimulation (Salmonella, $3 \mathrm{MOI}, 1 \mathrm{~h}$ ). (B) Co-IP analysis of polyubiquitination of endogenous NLRP3, AIM2, and NLRC4 in Lyz2-Cre-expressing Huwe $1^{+/ /}$and Huwe $7^{f / /}$ BMDMs stimulated with LPS $(500 \mathrm{ng} / \mathrm{mL}$, $4 \mathrm{~h}$ ) and ATP ( $5 \mathrm{mM}, 10 \mathrm{~min}$ ) or infected with $F$. novicida $(100 \mathrm{MOI}$, $12 \mathrm{~h}$ ) or Salmonella (3 MOI, $1 \mathrm{~h}$ ) as indicated. Data are representative of 3 independent experiments.

and exhibited distribution of large puncta after LPS and ATP simulation (Figure 8B). In addition, AIM2, NLRC4, and HUWE1 also formed large puncta within the BMDMs after dsDNA transfection, F. novicida infection, and Salmonella infection, respectively (Supplemental Figure 5, A and B).

ASC speck formation is a readout for inflammasome activation (39). Remarkably, ASC specks were frequently detected in Lyz2-Cre-expressing Huwe $1^{+/ Y}$ BMDMs in response to LPS plus ATP stimulation, dsDNA transfection, or infection with $F$. novicida or Salmonella but were rarely detected in Lyz2-Cre-expressing Huwe $1^{f / Y}$ BMDMs in response to those stimuli (Figure $8 \mathrm{C}$ ). Recently, the dominant species of active caspase- 1 induced by inflammasome activation in macrophages was shown to be fulllength $\mathrm{p} 46$ and a transient p33/p10 dimer; p33/p10 further carries out self-cleavage to release p20/p10, thereby terminating inflammasome activity (40). To examine how HUWE1 mediates caspase- 1 activation, we performed co-IP analysis of caspase- 1 and ASC in untreated and stimulated BMDMs. Interaction between ASC and cleaved caspase-1 p33 was induced in Lyz2Cre-expressing $H u w e 1^{+/ Y}$ BMDMs after stimulation, whereas this interaction was substantially reduced in the absence of HUWE1 (Figure 8D). Taken together, these data indicate that the ubiquiti- nation of NLRP3, AIM2, and NLRC4 mediated by HUWE1 promotes the assembly of inflammasomes and ASC speck formation, which increase the duration of caspase- 1 activation.

HUWE1 increases host defense against Salmonella, F. novici$d a$, and A. baumannii infection. Inflammasome activation plays important roles in host defense against bacterial infection; for example, the NLRC4 inflammasome contributes to host defense against Salmonella infection, the AIM2 inflammasome contributes to host defense against $F$. novicida infection, and the NLRP3 inflammasome contributes to host defense against $A$. baumannii and gram-negative bacterial infection $(1,29,41)$. To investigate the role of HUWE1 in host defense against bacterial infection, we treated age- and sex-matched $\mathrm{Huwe}^{\mathrm{I}^{+/+}}$-CreER and Huwe $1^{\mathrm{fl} / \mathrm{f}_{-}}$ CreER mice with tamoxifen for 5 consecutive days to induce Huwe1 gene deletion. Five days after the last injection of tamoxifen, the mice were infected with bacterial pathogens to analyze host defense and immune responses. Tamoxifen-treated Huwe $1^{+/+}$-CreER and Huwe $1^{f l / f l}$-CreER mice were intraperitoneally infected with Salmonella $\left(5.0 \times 10^{3} \mathrm{CFU}\right.$ per mouse), and body weight loss was monitored over time. Remarkably, we observed that tamoxifen-treated $H u w e 1^{f / f l}$ mice lost more body weight than did Huwe $^{+/+}$mice $(8.9 \%$ vs. $-6.5 \%$ of starting body weight, respec- 
A

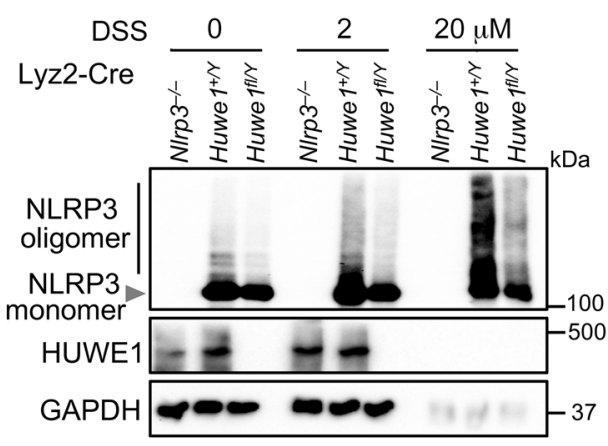

Lyz2-Cre ASC Speck
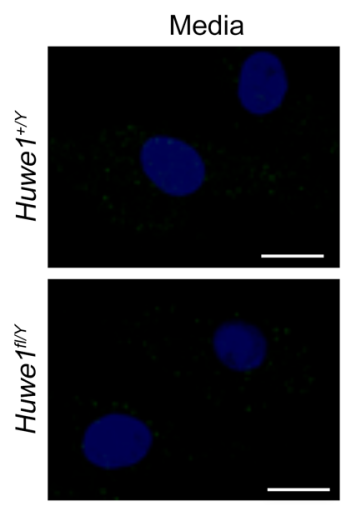

LPS + ATP
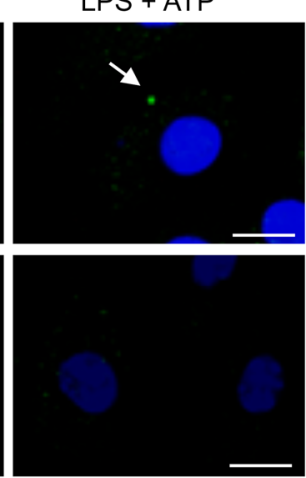

B
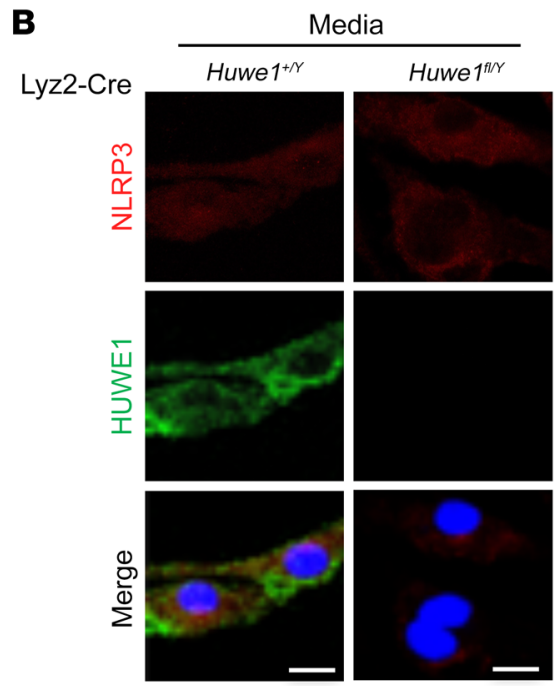

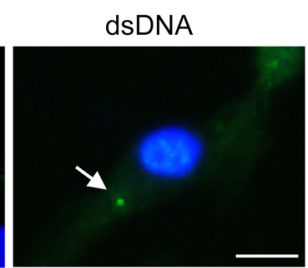

F. novicida
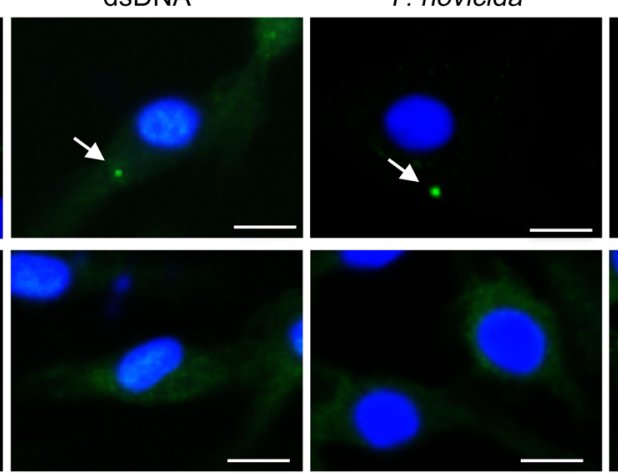

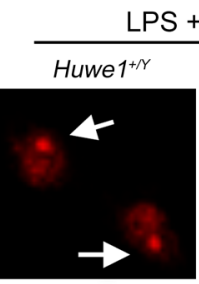

LPS + ATP
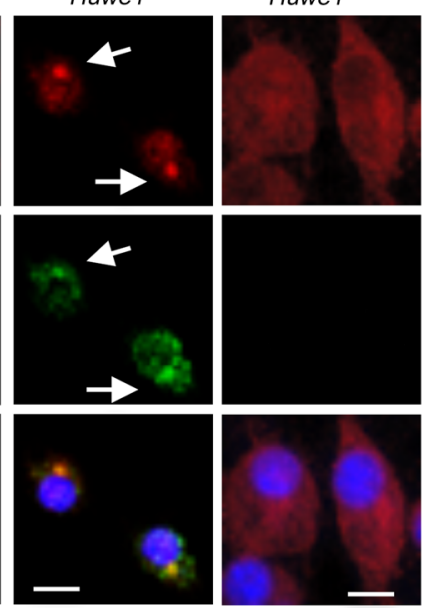

Salmonella
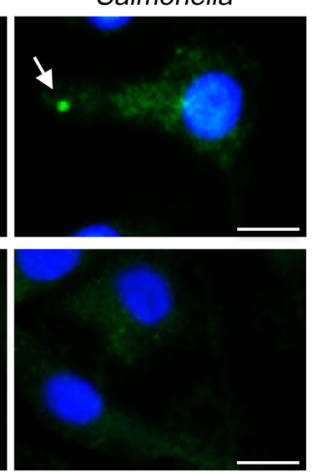

D

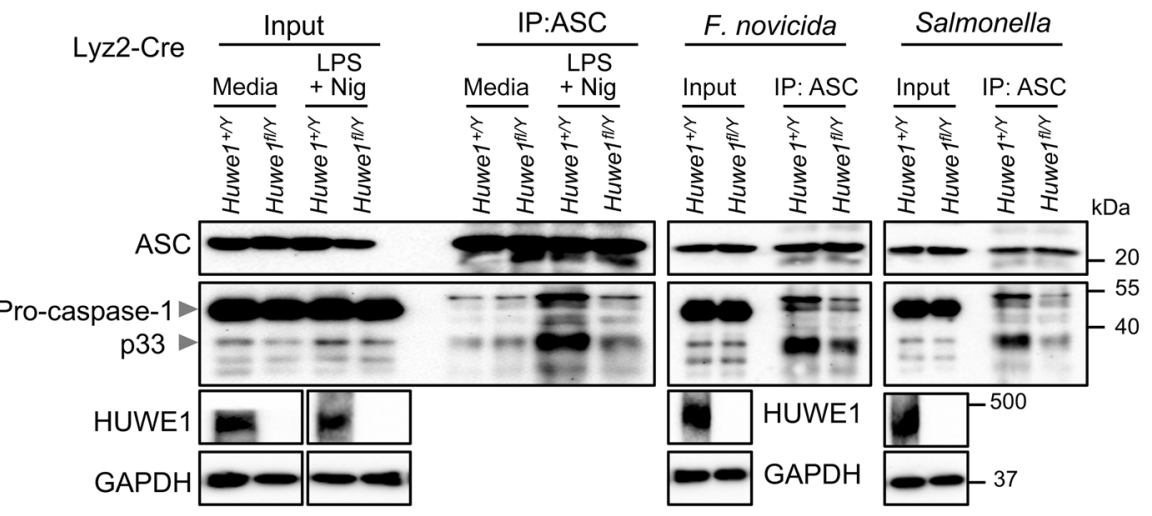

Figure 8. HUWE1 promotes inflammasome assembly and ASC speck formation. (A) Immunoblot analysis of NLRP3 oligomerization in N/rp3 $3^{-1-}$ and Lyz2-Creexpressing Huwe ${ }^{+/ / \gamma}$ and Huwe $7^{f / \gamma}$ BMDMs treated with DSS for 30 minutes at the indicated concentrations. (B) Confocal microscopic analysis of NLRP3 and HUWE1 in Lyz2-Cre-expressing Huwe ${ }^{+/ Y}$ and Huwe ${ }^{f / /}$ BMDMs without treatment or stimulated with LPS (500 ng/mL, 4 h) and ATP (5 mM, 20 min). Arrows indicate the distribution of large puncta. Scale bars: $10 \mu \mathrm{m}$. (C) Confocal microscopic analysis of ASC speck formation in Lyz2-Cre-expressing Huwe ${ }^{+/ \gamma}$ and Huwe ${ }^{f / Y}$ BMDMs without treatment or stimulated with LPS ( $\left.500 \mathrm{ng} / \mathrm{mL}, 4 \mathrm{~h}\right)$ and ATP (5 mM, $\left.20 \mathrm{~min}\right)$; or transfected with dsDNA (1.5 $\left.\mu \mathrm{g}, 30 \mathrm{~min}\right) ;$ or infected with F. novicida (100 MOI, 12 h) or Salmonella (3 MOI, 1 h) as indicated. Arrows indicate ASC specks. Scale bars: $10 \mu \mathrm{m}$. (D) Co-IP analysis of caspase-1 that coim-

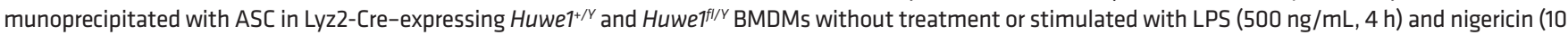
$\mu \mathrm{g} / \mathrm{mL}, 30 \mathrm{~min}$ ) or infected with F. novicida (100 MOI, $12 \mathrm{~h}$ ) or Salmonella (3 MOI, $1 \mathrm{~h}$ ) as indicated. Data are representative of 3 independent experiments.

tively) (Figure 9A). We counted bacteria in the spleens and livers of $\mathrm{Huwe}^{+/ /+}$and Huwe ${ }^{f / / f l}$ mice after 2 days of infection with Salmonella. The bacterial burdens in the spleens and livers of Huwe $1^{f / f l}$ mice were significantly higher than those in the spleens and livers of $\mathrm{Huwe}^{+/+}$mice (Figure 9B). H\&E staining revealed more inflammation and immune cell infiltration in the livers of infected $H u w e 1^{f l / l}$ mice than in those of $H u w e 1^{+/+}$mice (Figure 9E). In the case of $F$. novicida infection-triggered AIM2 inflammasome activation, tamoxifen-treated $H u w e 1^{+/+}$and $H u w e 1^{f l / f l}$ mice were subcutaneously infected with $F$. novicida $\left(1.5 \times 10^{5} \mathrm{CFU}\right.$ per mouse), 
and mouse morbidity was monitored. In line with the Salmonella infection data, we found that tamoxifen-treated $H u w e 1^{f / f l}$ mice lost more body weight than did $\mathrm{Huwe}^{+/+}$mice $(7.7 \%$ vs. $3.7 \%$ of starting body weight, respectively) (Figure 9C). The bacterial burdens in the livers and spleens of $H u w e 1^{f l / l}$ mice were significantly higher than those in $\mathrm{Huwe}^{1^{+/+}}$mice (Figure 9D). The difference in immune cell infiltration induced by $F$. novicida infection in the livers of infected Huwe ${ }^{f l f l}$ mice compared with $\mathrm{Huwe}^{+/+}$mice was revealed by H\&E staining (Figure 9E). To determine whether NLRP3 inflammasome activation mediated by HUWE1 is involved in the host defense against gram-negative bacterial infection, tamoxifen-treated $\mathrm{Huwe}^{+/ /+}$and $\mathrm{Huwe} \mathrm{1}^{\mathrm{fl} / \mathrm{fl}}$ mice were intranasally infected with $A$. baumannii $\left(5.0 \times 10^{8} \mathrm{CFU}\right.$ per mouse), and the bacterial burden was assessed. We found that the bacterial load in the lungs and immune cell infiltration into the lungs of Huwe $1^{f / f l}$ mice were significantly higher than in $\mathrm{Huwe}^{+/ /+}$mice (Figure 9, F and G).

We analyzed the activation of caspase- 1 in vivo in Salmonellainfected liver and spleen, F. novicida-infected liver, and A. baumannii-infected lung tissues. Notably, we observed that caspase-1 activation (as shown by blotting for p20) triggered by bacterial infection was dramatically reduced in $H u w e 1^{f / / f l}$ mice compared with that seen in $\mathrm{Huwe}^{1^{+/+}}$mice (Figure 9, H and I). Consistent with the caspase-1 activation data, the level of IL-1 $\beta$ in the sera of Huwe $1^{f / f l}$ mice was significantly lower than that in the sera of $\mathrm{Huwe}^{+/+}$mice upon $F$. novicida, Salmonella, and A. baumannii infection (Figure 9J). Instead, the production of TNF and IL-6 after bacterial infection was comparable between $\mathrm{Huwe}^{+/+}$and $\mathrm{Huwe} \mathrm{f}^{f / / l}$ mice (Figure 9J), suggesting that HUWE1 specifically mediates inflammasome activation in response to bacterial infection. In line with the data on the HUWE1-deficient mice, administration of the HUWE1 inhibitor BI8622 significantly increased the bacterial burden and reduced caspase-1 activation in vivo upon Salmonella infection (Supplemental Figure 6, A and B). These data collectively indicate that HUWE1 regulates inflammasome activation and host defense against bacterial infection through mediation of the K27-linked polyubiquitination of NLRP3, AIM2, and NLRC4, which contributes to sustained inflammasome activation (Supplemental Figure 7).

\section{Discussion}

Inflammasome activation and ubiquitination are 2 major host innate immune events during bacterial infection $(12,42)$. Both extracellular and intracellular bacterial pathogens are able to trigger different types of inflammasome activation through their engagement with diverse cytosolic receptors, such as NLRP3, AIM2, and NLRC4. Similar to the ubiquitination-mediated NF-אB and type I IFN signaling pathways, ubiquitination-mediated inflammasome activation is increasingly recognized, adding an additional layer of complexity to the regulation of inflammasome activation. Instead, the molecular mechanisms by which ubiquitination mediates inflammasome assembly and the activation of caspase- 1 remain largely unknown (12). Our study demonstrates that the cytosolic receptors NLRP3, AIM2, and NLRC4 are simultaneously regulated by one E3 ubiquitin ligase, HUWE1. HUWE1-mediated K27-linked polyubiquitination of NLRP3, AIM2, and NLRC4 was shown to be essential for inflammasome assembly and caspase-1 activation. The molecule MCC950, which directly targets NLRP3 and inhibits NLRP3 inflammasome activation, has potential clinical applica- tions (43). As ubiquitination is a reversible process, modulation of the ubiquitination process is an attractive therapeutic approach for specifically targeting aberrant inflammasome activation. Thus, the HUWE1-specific inhibitor BI8622 shows great potential for treating excessive NLRP3, AIM2, and NLRC4 inflammasome activation-triggered inflammatory diseases.

Inflammasome activation is a double-edged sword for intracellular bacterial pathogens, as it triggers an inflammatory response but simultaneously causes pyroptotic cell death, reducing the replication niche of intracellular bacteria. Thus, the assembly and activation of inflammasomes need to be tightly regulated at multiple levels during bacterial infection. The positive role of HUWE1 in host defense against different bacterial infections paves the way to the regulation of inflammasome activity and host defense through modulation of the host ubiquitin machinery. Our data showed that HUWE1 interacts with NLRP3, AIM2, and NLRC4 mainly through a $\mathrm{BH} 3$ domain-containing fragment, which is consistent with previous findings that $\mathrm{BH} 3$ is essential for protein interaction $(44,45)$. HUWE1 might have evolved a conserved domain to directly interact with diverse pattern recognition receptors (PRRs) and function as a master regulator, mediating the activation of multiple inflammasomes. A conformational change was shown to be important for the activity of HUWE1 (46). How the activity of HUWE1 is regulated in response to distinct bacterial pathogens needs to be further explored, and whether it is effective against bacterial infection through the boosting of HUWE1 activity remains an intriguing question.

Inflammasome formation is coordinated by cytosolic PRRs, such as NLRP3, AIM2, and NLRC4, in response to various sterile and pathogenic stimuli. PRRs interact with pro-caspase-1 with or without the adapter protein ASC to form a platform for caspase-1 activation (30). Although numerous studies have shown the roles of inflammasome activation and function in development and disease progression, the detailed mechanisms of inflammasome assembly and caspase-1 activation remain unclear (40). A hallmark of caspase- 1 activation is the processing of pro-caspase- 1 into its p20 and p10 subunits, which have long been presumed to be the primary active enzymes. Recently, the dominant form of active caspase-1 was reported to be full-length p46 and a transient p33/ p10 dimer, whereas formation of the p20/p10 dimer was found to reflect the inactivation of caspase- $1(40,47,48)$. Our results indicate that HUWE1 preferentially mediates the interaction between ASC and the $\mathrm{p} 33$ form of cleaved caspase- 1 and promotes caspase- 1 activation, which is consistent with recent findings. A previous study demonstrated that linear ubiquitination-mediated ASC oligomerization is essential for inflammasome complex formation (49). Our work has revealed that the K27-linked polyubiquitination of NLRP3 is important for its oligomerization and inflammasome assembly. We hypothesize that HUWE1-mediated K27-linked polyubiquitination of NLRP3, AIM2, and NLRC4 produces a scaffold to maintain ASC speck formation and increase the interaction between ASC and cleaved caspase-1.

HUWE1, also known as MULE or ARF-BP1, a large protein with a molecular weight close to $500 \mathrm{kDa}$, modulates a wide array of cellular functions through the targeting of a number of substrates for ubiquitination, such as N-Myc, Cdc6, p53, and histones involved in the cell cycle, stem cell lineage commitment, and 

CreER tamoxifen

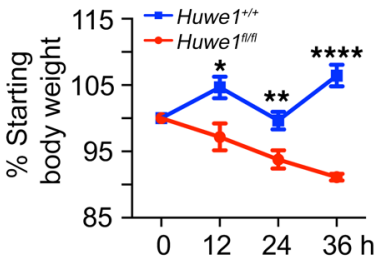

F. novicida

C CreER tamoxifen

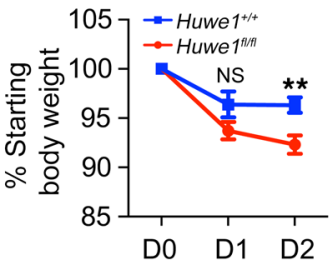

A Samonella

B

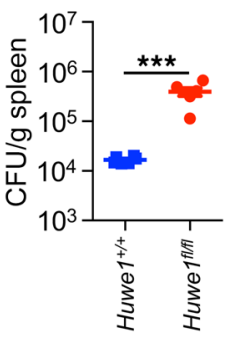

D

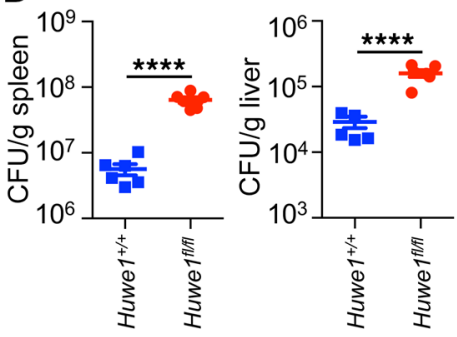

$F$

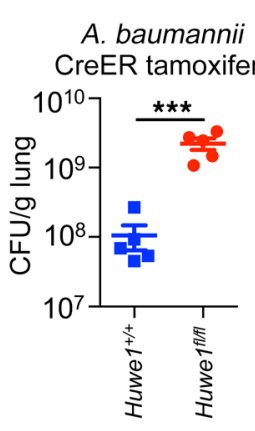

\section{G}
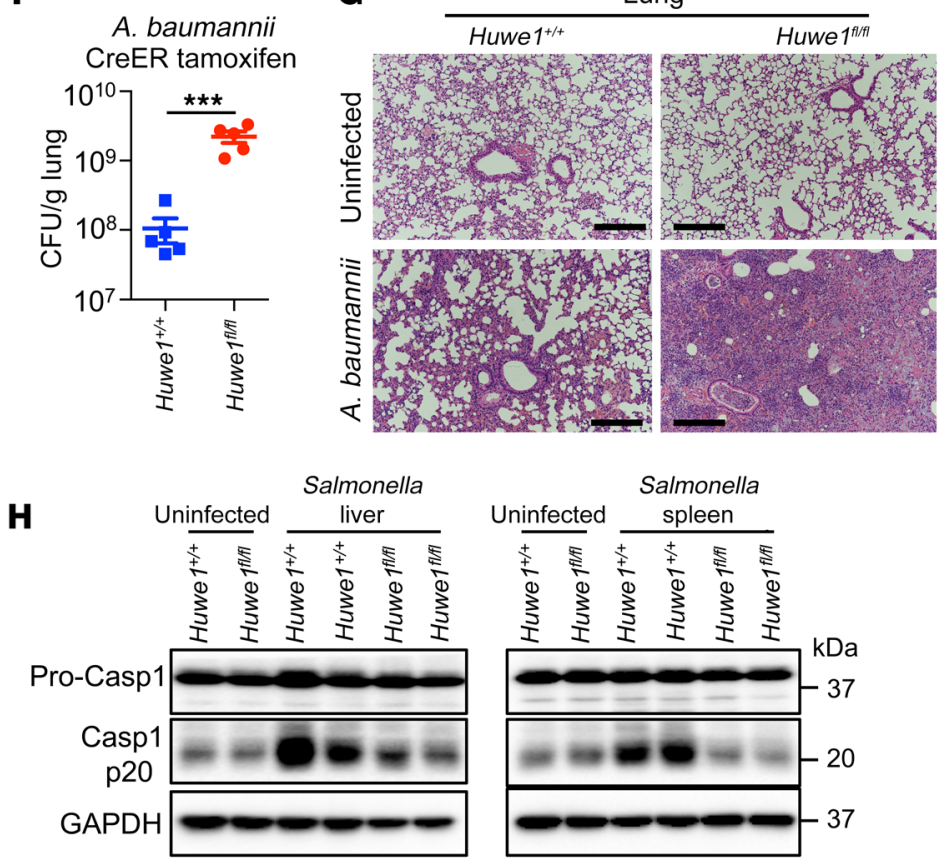

F. novicida

I

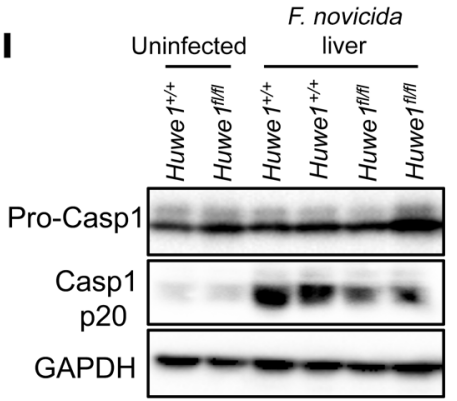

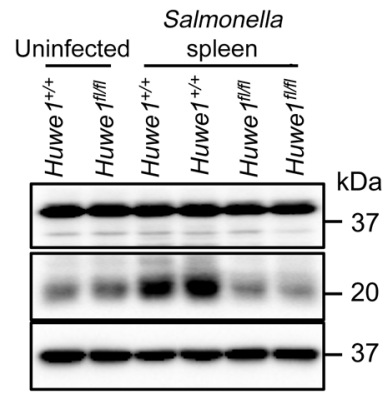

A. baumannii

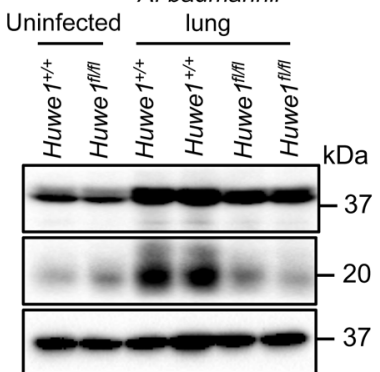

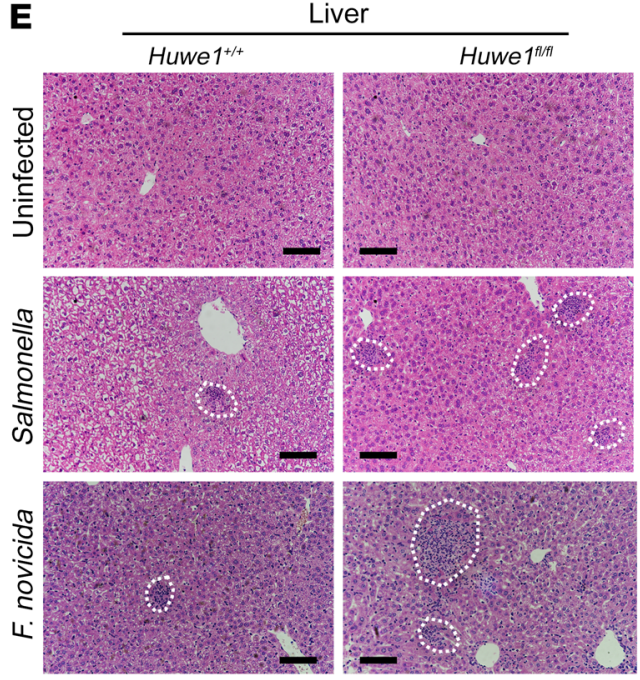

J

CreER tamoxifen sera
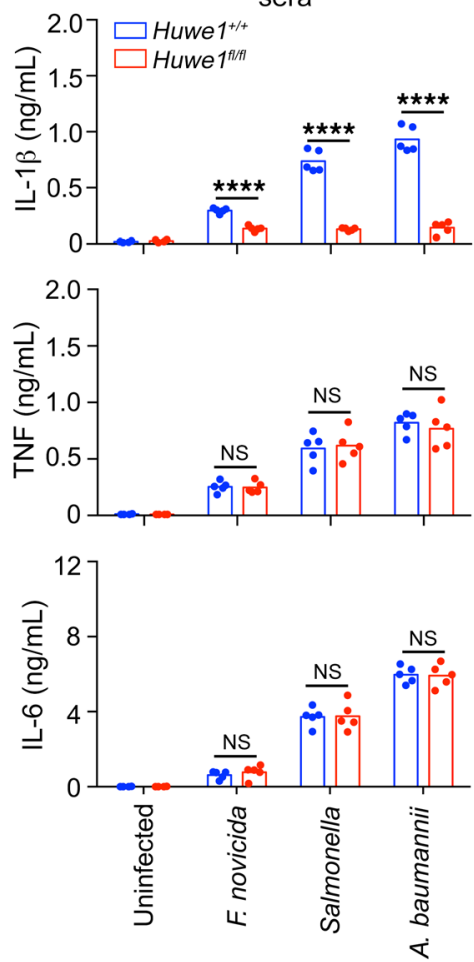
Figure 9. HUWE1 deficiency increases host susceptibility to Salmonella, F. novicida, and $A$. baumannii infection. Huwe $7^{f / f / 1}-$ CreER and Huwe ${ }^{+/++}$ CreER mice were injected with tamoxifen ( $2 \mathrm{mg} / 100 \mu \mathrm{L}$ per mouse) for 5 consecutive days. Five days after the last injection, mice were infected with bacterial pathogens, and the bacterial burden and host immune responses were analyzed. (A and B) Tamoxifen-treated Huwe fl/fI-CreER

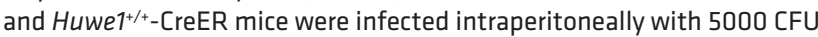
Salmonella, and body weight change after infection (A) and bacterial burden in the spleen and liver on day 2 after infection (B) were measured. (C

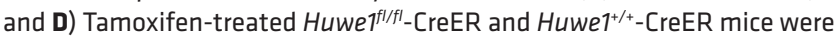
infected subcutaneously with $1.5 \times 10^{5} \mathrm{CFU} F$. novicida, and (C) body weight change after infection and (D) bacterial burden in the spleen and liver on day 2 after infection were measured. (E) H\&E staining of liver sections from uninfected and Salmonella- or F. novicida-infected mice in $\mathbf{B}$ and $\mathbf{D}$. Dashed outlines indicate infiltrated immune cells. Scale bars: $100 \mu \mathrm{m}$. (F) Tamoxifen-treated Huwe $7^{f / / f l}-\mathrm{CreER}$ and Huwe ${ }^{{ }^{+/+}-\mathrm{CreER}}$ mice were infected intranasally with $5.0 \times 10^{8} \mathrm{CFU} A$. baumannii, and bacterial burden in the lungs on day 1 after infection was measured. (C) H\&E staining of lung sections from uninfected and $A$. baumannii-infected mice in $\mathbf{F}$. Scale bars: $100 \mu \mathrm{m}$. (H and I) Immunoblot analysis of pro-caspase-1 and its subunit p20 in the liver, spleen, and lungs of uninfected, Salmonella-infected (H), $F$. novicida-infected, and $A$. baumannii-infected (I) mice. GAPDH was used as a loading control. (J) ELISA analysis of IL-1B, TNF, and IL-6 in sera from uninfected and bacteria-infected mice in $\mathbf{B}, \mathbf{D}$, and $\mathbf{F}$. Each dot represents an individual mouse (B, D, F, and J). ${ }^{*} P<0.05,{ }^{* *} P<0.01,{ }^{* *} P<0.001$, and ${ }^{* * *} P<0.0001$, by 2 -sided Student's $t$ test without multiple-comparisons correction. Data are representative of 2 independent experiments.

tumorigenesis (50-54). HUWE1 also plays important roles in regulating the DNA damage response (DDR) by targeting histones, MCL-1, and Chk1 $(44,45,55,56)$. In addition, clinical evidence and exome sequencing revealed that HUWE1 mutations cause $\mathrm{X}$ chromosome-linked intellectual disability (57-59). HUWE1 is also thought to have sex-specific effects on susceptibility to infectious diseases, although the detailed mechanism is unknown (60). Given the strong association between inflammation and the pathogenesis of various diseases, our finding that HUWE1 mediates inflammasome activation indicates that HUWE1 acts as an unexpected, alarming initiator of diseases and insults. Our study offers insights into the mechanisms of HUWE1-associated diseases and provides a potential therapeutic target for the treatment of inflammasome dysregulation-mediated disorders.

\section{Methods}

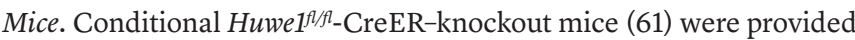
by X. Zhao (Sichuan University, Chengdu, China); Aim2 ${ }^{--}$mice were provided by S. Feng (National Institute of Biological Sciences, Beijing, China); and $\mathrm{Nlrp3}^{-/}$(62) mice were provided by D. Wang (Zhejiang University, Hangzhou, China). WT and knockout mice were kept under specific pathogen-free conditions in the Animal Resource Center at Kunming Institute of Zoology, Chinese Academy of Sciences.

Bacterial infection of mice. The bacterial strains used in this study included the F. novicida strain U112, Listeria monocytogenes, Salmonella typhimurium, and A. baumannii and were grown as previously described $(41,63)$.

Eight- to 10-week-old, sex-matched $\mathrm{Huwe}^{1^{+/+(+/ \gamma)}-\mathrm{CreER} \text { and }}$ Huwe $^{f / f(f(t) / Y)}$-CreER mice were injected with tamoxifen $(2 \mathrm{mg} / 100$ $\mu \mathrm{L}$ sunflower seed oil per mouse) for 5 consecutive days as previously described (64). Tamoxifen-treated mice were infected intraperitoneally with $S$. typhimurium (5000 CFU per mouse), subcutaneously with
F.novicida U112 $\left(1.5 \times 10^{5} \mathrm{CFU}\right.$ per mouse $)$, or intranasally with $A$. baumannii $\left(5.0 \times 10^{8} \mathrm{CFU}\right.$ per mouse), as indicated. Mice were weighed and monitored daily over a 2-day period. Mice were euthanized on the indicated postinfection days (Figure 9, B, D, and F), and livers, spleens, and lungs were harvested to determine the bacterial burden.

Preparation of BMDMs, stimulation, and bacterial infection. To generate BMDMs, bone marrow (BM) cells were cultured in L929 cell-conditioned DMEM/F-12 supplemented with 10\% FBS, 1\% nonessential amino acids, and $1 \%$ penicillin-streptomycin for 5 days as previously described. Huwe1 gene deletion in BMDMs was performed by adding 4-OHT (100 nM) for 5 days. The HUWE1 inhibitor BI8622 (HY-120929) was purchased from MedChemExpress. BMDMs were treated with BI8622 $(10 \mu \mathrm{M})$ for 2 hours before stimulation and infection. Control and treated BMDMs were stimulated with ligands or infected with bacterial pathogens for the indicated durations as previously described (63). The treated and control cells were lysed for RNA and protein analysis.

Immunoblot analysis and antibodies. Samples were separated by $12 \%$ SDS-PAGE, followed by electrophoretic transfer to polyvinylidene fluoride membranes, and membranes were blocked and then incubated with primary antibodies. The following primary antibodies were used: anti-V5 (Cell Signaling Technology [CST], 13202); anti-AIM2 (CST, 13095); anti-caspase-1 (AdipoGen, AG-20B-0042); anti-HUWE1 (Lifespan Biosciences, LS-B1359); anti-NLRP3 (AdipoGen, AG-20B-0014); anti-NLRC4 (Lifespan Biosciences, LS-C148271); anti-ASC (AdipoGen, AG-25B-0006); anti-HA (Santa Cruz Biotechnology, SC-805); antiFLAG (MilliporeSigma, F3165); anti-ubiquitin (MilliporeSigma, 04-263); anti-Myc (CST, 2278); and anti-GAPDH (CST, 5174). HRP-labeled anti-rabbit, anti-mouse, or anti-goat (CST) was used as the secondary antibody. ImageJ software (NIH) was used for signaling quantification.

Immunofluorescence staining and microscopy. For ASC, NLRP3, AIM2, and NLRC4 immunostaining, infected and uninfected BMDMs were fixed in $4 \%$ paraformaldehyde for 15 minutes at room temperature. Cells were washed with PBS and blocked in $1 \times$ ELISA buffer with $0.1 \%$ saponin for 1 hour. Cells were stained with anti-ASC (AdipoGen, AG-25B-0006); anti-NLRP3 (AdipoGen, AG-20B-0014); anti-AIM2 (CST, 13095); anti-NLRC4 (LifeSpan Biosciences, LS-C148271); antiTGN46 (Abcam, ab2809); or anti-HUWE1 (LifeSpan Biosciences, LS-B1359) - all at 1:300 to 1:500 dilution, overnight at $4^{\circ} \mathrm{C}$. Cells were washed, stained with a fluorescence-conjugated secondary antibody for 40 minutes at $37^{\circ} \mathrm{C}$, and mounted using mounting medium (Vector Laboratories, H-1200). Cells were observed on the Zeiss LSM880 confocal microscope, and image acquisition and data analysis were performed using Zeiss ZEN black_2-3SP1 and ZEN blue 2.6 software.

$I P-M S$ analysis. WT and Aim2-- BMDMs were infected with F. novicida for 12 hours and lysed in IP buffer. AIM2 antibody was used for IP. The MS experiment and data processing were performed by Novogene Company. Proteins that were specifically identified in WT BMDMs, but not in the Aim2 ${ }^{-/}$BMDMs, are listed in Supplemental Table 1.

Plasmid transfection and co-IP experiments. The plasmids expressing full-length WT and C4341A-mutated HUWE1 were provided by X. Zhao (Sichuan University, Chengdu), and truncated HUWE1 plasmids were provided by G. Shao (Peking University, Beijing) as previously described (44, 61, 65). Aim2, Nlrp3, Nlrc4, Asc, and caspase-1 were amplified from a mouse cDNA library and subcloned into $\mathrm{pCDH}, \mathrm{pCMV}$, and $\mathrm{MSCV}$ vectors. Truncated DNA sequences were amplified from full-length of cDNA plasmids and subcloned into $\mathrm{PCDH}$ vector. Site-directed mutations were generated using QuikChange site-directed mutagenesis kits. 
All plasmids were confirmed by DNA sequencing. The primer sequences for vector construction are listed in Supplemental Table 2. Lipofectamine 3000 reagents (Invitrogen, Thermo Fisher Scientific) were used for transient transfection of plasmids into HEK293T cells.

For IP, whole HEK293T cells collected 36 hours after transfection or BMDMs with and without treatments were lysed in IP buffer composed of $50 \mathrm{mM}$ Tris- $\mathrm{HCl}$ (pH 7.4), 2 mM EDTA, $150 \mathrm{mM} \mathrm{NaCl}$, $1 \%$ NP-40, and protease-phosphatase inhibitor cocktails (BioTools). After centrifugation, supernatants were collected and incubated with protein A/G Plus-Agarose (Santa Cruz Biotechnology, sc-2003) and $3 \mu \mathrm{g}$ of the corresponding antibodies for 12 hours at $4^{\circ} \mathrm{C}$, followed by 5 washes with IP buffer. Anti-HUWE1 (Abcam, ab70161) was used for endogenous co-IP analysis. Immunoprecipitated components were eluted by boiling in the SDS loading buffer for 10 minutes. For immunoblot analysis, immunoprecipitates and input lysates were separated by SDS-PAGE, followed by transfer onto PVDF membranes and detection by specific antibodies.

Ubiquitination analysis. Ubiquitin-expressing plasmids were provided by C. Chen (Kunming Institute of Zoology, CAS, Kunming, China). For polyubiquitination analysis in HEK293T cells, HEK293T cells were transfected with plasmids expressing HUWE1, HA-ubiquitin (WT), or HA-ubiquitin (K63, K6, K11, K27, mutant K27R, K29, K33, or K48) with MG132, and Flag-AIM2, NLRP3, or NLRC4 (WT, truncated, and site-directed mutants). For ubiquitination analysis of NLRP3, AIM2, and NLRC4 in BMDMs, Lyz2-Cre-expressing Huwe ${ }^{+/ Y}$ and Lyz2-Creexpressing Huwe ${ }^{f / / Y}$ BMDMs were treated with LPS and ATP or infected with Salmonella or F. novicida for the indicated durations. Transfected HEK293T cells and treated BMDMs were lysed in lysis buffer $(50 \mathrm{mM}$ Tris-HCl [pH 6.8], 1.5\% SDS) and then boiled for 15 minutes. The denatured samples were immunoprecipitated with the anti-FLAG and specific primary antibodies, and the IP products were analyzed by immunoblotting with anti-HA, anti-ubiquitin, and specific antibodies.

DSS cross-linking. BMDMs were treated as indicated above and then harvested and washed with cold PBS. Cell pellets were lysed in HEPES lysis buffer (30 mM HEPES [pH7.4],150 mM NaCl,1\% NP-40, and protease inhibitor cocktails) for 30 minutes at $4^{\circ} \mathrm{C}$. Cell lysates were centrifuged at $13,000 \mathrm{~g}$ for 10 minutes at $4^{\circ} \mathrm{C}$. The lysates were then cross-linked with DSS (at the concentrations indicated in Figure $8 \mathrm{~A})$ for 30 minutes at room temperature. The reaction was quenched with $50 \mathrm{mM}$ Tris- $\mathrm{HCl}$ (pH7.5) for 15 minutes. The cleared, cross-linked samples were boiled in SDS loading buffer and analyzed by immunoblotting with specific antibodies.

Lentivirus production and infection. The viral particles were prepared by transfecting HEK293T cells with caspase-1- and AIM2expressing plasmids in combination with packaging vectors. Twelve hours later, the media were replaced with fresh complete DMEM. Viral supernatant was harvested and passed through a $0.45 \mu \mathrm{m}$ syringe filter 48 and 72 hours after transfection. To establish stably infected cells, HEK293T cells were infected 3 times with filtered lentiviral supernatant in the presence of polybrene $(8 \mu \mathrm{g} / \mathrm{mL})$. The infected cells were selected by puromycin $(1 \mu \mathrm{g} / \mathrm{mL})$ for 7 days, and the surviving cells were cultured in fresh media.
Preparation of tissue samples for HE staining. The superior lobes of the right lung and liver were fixed in $10 \%$ formalin, and $5 \mu \mathrm{m}$ sections were stained with H\&E and examined with a microscope.

Real-time qRT-PCR. Total RNA was isolated from cells and tissues using TRIzol Reagent (Invitrogen, Thermo Fisher Scientific). cDNA was reverse transcribed using M-MLV Reverse Transcriptase (Promega). Real-time quantitative reverse transcription PCR (qRT-PCR) was performed on the Bio-Rad CFX-96 Touch Real-Time Detection System. The primer sequences are listed in Supplemental Table 2.

ELISA. The in vivo and in vitro samples were analyzed for cytokine release using ELISA MAX Standard Sets from BioLegend (mouse IL-1 $\beta$, 432601; mouse IL-6, 431301; mouse TNF, 430901; human IL-1, 437004; human IL-6, 430504; human TNF, 430204) according to the manufacturer's instructions.

Statistics. Data are presented as the mean \pm SEM. Statistical analyses were performed using 2-tailed Student's $t$ and log-rank tests. $P$ values of 0.05 or less were considered significant.

Study approval. All animal experiments were conducted in accordance with guidelines approved by the IACUC of the Kunming Institute of Zoology, Chinese Academy of Sciences. The present study was approved by the IRB of the Kunming Institute of Zoology, Chinese Academy of Sciences.

\section{Author contributions}

XQ, YG, LL, and TX designed the study. YG, LL, TX, XG, CW, YL, YY, DY, BS, XZ, GS, and XQperformed experiments and analyzed the data. XQ and TX wrote the manuscript. YG, LL, TX, and XG contributed equally to data analysis. XQ conceived the study and provided overall direction. The order of co-first authorship was determined by the authors' contributions to data acquisition and drafting of the manuscript.

\section{Acknowledgments}

We thank X. Zhao (Sichuan University, Chengdu, China) for providing Huwe1 conditional-knockout mice and HUWE1 WT and C4341A-mutant plasmids; G. Shao (Peking University, Beijing, China) for the HUWE1 truncated plasmids; C. Chen (Kunming Institute of Zoology, CAS, Kunming, China) for ubiquitin plasmids and Lyz2-Cre mice; F. Shao (National Institute of Biological Sciences, Beijing, China) for Aim2 $2^{-/}$mice; and D. Wang (Zhejiang University, Hangzhou, China) for the $\mathrm{Nlrp3}^{-/-}$mice. This work was supported by the National Key Research and Development Program of China (2017YFD0500300); the National Natural Science Foundation of China (31970896, 31701134, 81701578, and 82072255); and Yunnan Province (2019FJ008, 2018FA038, 2018FB127, 2018FB131, HXDTZX-2019-1, HXDT2019-2, and AMHD-2018-2).

Address correspondence to: Xiaopeng Qi, Kunming Institute of Zoology, Chinese Academy of Sciences, 32 East Jiaochang Road, Kunming, Yunnan 650223, China. Phone: 86.871.65110289; Email: qixiaopeng@mail.kiz.ac.cn.
1. Latz E, Xiao TS, Stutz A. Activation and regulation of the inflammasomes. Nat Rev Immunol. 2013;13(6):397-411.

2. Lamkanfi M, Dixit VM. Mechanisms and functions of inflammasomes. Cell. 2014;157(5):1013-1022.

3. Davis BK, Wen H, Ting JP. The inflammasome NLRs in immunity, inflammation, and associated diseases. Annu Rev Immunol. 2011;29:707-735.
4. Guo H, Callaway JB, Ting JP. Inflammasomes: mechanism of action, role in disease, and therapeutics. Nat Med. 2015;21(7):677-687.

5. Saitoh T, Akira S. Regulation of inflam- 
masomes by autophagy. J Allergy Clin Immunol. 2016;138(1):28-36.

6. Shim DW, Lee KH. Posttranslational regulation of the NLR family pyrin domain-containing 3 inflammasome. Front Immunol. 2018;9:1054.

7. Bednash JS, Mallampalli RK. Regulation of inflammasomes by ubiquitination. Cell Mol Immunol. 2016;13(6):722-728.

8. Kattah MG, Malynn BA, Ma A. Ubiquitin-modifying enzymes and regulation of the inflammasome. J Mol Biol. 2017;429(22):3471-3485.

9. Barry R, et al. SUMO-mediated regulation of NLRP3 modulates inflammasome activity. Nat Commun. 2018;9(1):3001.

10. Maculins T, Fiskin E, Bhogaraju S, Dikic I. Bacteria-host relationship: ubiquitin ligases as weapons of invasion. Cell Res. 2016;26(4):499-510.

11. Shi C-S, et al. Activation of autophagy by inflammatory signals limits IL- $1 \beta$ production by targeting ubiquitinated inflammasomes for destruction. Nat Immunol. 2012;13(3):255-263.

12. Xu T, Guo Y, Qi X. Ubiquitination-mediated inflammasome activation during bacterial infection. Int J Mol Sci. 2019;20(9):E2110.

13. Py BF, Kim MS, Vakifahmetoglu-Norberg H, Yuan J. Deubiquitination of NLRP3 by BRCC3 critically regulates inflammasome activity. Mol Cell. 2013;49(2):331-338.

14. Palazón-Riquelme P, et al. USP7 and USP47 deubiquitinases regulate NLRP3 inflammasome activation. EMBO Rep. 2018;19(10):e44766.

15. Lee JY, et al. The deubiquitinating enzyme, ubiquitin-specific peptidase 50, regulates inflammasome activation by targeting the ASC adaptor protein. FEBS Lett. 2017;591(3):479-490.

16. Yan Y, et al. Dopamine controls systemic inflammation through inhibition of NLRP3 inflammasome. Cell. 2015;160(1-2):62-73.

17. Song $\mathrm{H}$, et al. The E3 ubiquitin ligase TRIM31 attenuates NLRP 3 inflammasome activation by promoting proteasomal degradation of NLRP3. Nat Commun. 2016;7:13727.

18. Kawashima A, et al. ARIH2 ubiquitinates NLRP3 and negatively regulates NLRP3 inflammasome activation in macrophages. J Immunol. 2017;199(10):3614-3622.

19. Han S, et al. Lipopolysaccharide primes the NALP3 inflammasome by inhibiting its ubiquitination and degradation mediated by the SCFFBXL2 E3 ligase. J Biol Chem. 2015;290(29):18124-18133.

20. Wan P, et al. Cullin1 binds and promotes NLRP3 ubiquitination to repress systematic inflammasome activation. FASEB J. 2019;33(4):5793-5807.

21. Humphries F, et al. The E3 ubiquitin ligase Pellino2 mediates priming of the NLRP3 inflammasome. Nat Commun. 2018;9(1):1560.

22. Guan K, et al. MAVS promotes inflammasome activation by targeting ASC for K63-linked ubiquitination via the E3 ligase TRAF3. JImmunol. 2015;194(10):4880-4890.

23. Xing Y, et al. Cutting edge: TRAF6 mediates TLR/IL-1R signaling-induced nontranscriptional priming of the NLRP3 inflammasome. J Immunol. 2017;199(5):1561-1566.

24. Sandstrom A, Mitchell PS, Goers L, Mu EW, Lesser CF, Vance RE. Functional degradation: a mechanism of NLRP1 inflammasome activation by diverse pathogen enzymes. Science. 2019;364(6435):eaau1330.

25. Chui AJ, et al. N-terminal degradation activates the NLRP1B inflammasome. Science. 2019;364(6435):eaau1208.

26. $\mathrm{Xu} \mathrm{H}$, et al. The $\mathrm{N}$-end rule ubiquitin ligase UBR2 mediates NLRP1B inflammasome activation by anthrax lethal toxin. $E M B O J$. 2019;38(13):e101996.

27. Man SM, et al. The transcription factor IRF1 and guanylate-binding proteins target activation of the AIM2 inflammasome by Francisella infection. Nat Immunol. 2015;16(5):467-475.

28. Rathinam VAK, et al. The AIM2 inflammasome is essential for host defense against cytosolic bacteria and DNA viruses. Nat Immunol. 2010;11(5):395-402.

29. Jones JW, et al. Absent in melanoma 2 is required for innate immune recognition of Francisella tularensis. Proc Natl Acad Sci USA. 2010;107(21):9771-9776.

30. Man SM, Kanneganti TD. Converging roles of caspases in inflammasome activation, cell death and innate immunity. Nat Rev Immunol. 2016;16(1):7-21.

31. Mariathasan S, et al. Differential activation of the inflammasome by caspase- 1 adaptors ASC and Ipaf. Nature. 2004;430(6996):213-218.

32. Kim S, et al. Listeria monocytogenes is sensed by the NLRP3 and AIM2 inflammasome. Eur $J$ Immunol. 2010;40(6):1545-1551.

33. Peter S, et al. Tumor cell-specific inhibition of MYC function using small molecule inhibitors of the HUWE1 ubiquitin ligase. EMBO Mol Med. 2014;6(12):1525-1541.

34. Chen J, Chen ZJ. PtdIns4P on dispersed transGolgi network mediates NLRP3 inflammasome activation. Nature. 2018;564(7734):71-76.

35. Roberts TL, et al. HIN-200 proteins regulate caspase activation in response to foreign cytoplasmic DNA. Science. 2009;323(5917):1057-1060.

36. Bürckstümmer T, et al. An orthogonal proteomicgenomic screen identifies AIM2 as a cytoplasmic DNA sensor for the inflammasome. Nat Immunol. 2009;10(3):266-272.

37. Hornung V, et al. AIM2 recognizes cytosolic dsDNA and forms a caspase-1-activating inflammasome with ASC. Nature. 2009;458(7237):514-518.

38. Fernandes-Alnemri T, Yu JW, Datta P, Wu J, Alnemri ES. AIM2 activates the inflammasome and cell death in response to cytoplasmic DNA. Nature. 2009;458(7237):509-513.

39. Stutz A, Horvath GL, Monks BG, Latz E. In: De Nardo CM, Latz E eds. The Inflammasome: Methods and Protocols. Humana Press; 2013:91-101.

40. Boucher D, et al. Caspase-1 self-cleavage is an intrinsic mechanism to terminate inflammasome activity. JExp Med. 2018;215(3):827-840.

41. Li Y, et al. Type I IFN operates pyroptosis and necroptosis during multidrug-resistant A. baumannii infection. Cell Death Differ. 2018;25(7):1304-1318

42. Lopez-Castejon G. Control of the inflammasome by the ubiquitin system. FEBS J. 2020;287(1):11-26.

43. Coll RC, et al. MCC950 directly targets the NLRP3 ATP-hydrolysis motif for inflammasome inhibition. Nat Chem Biol. 2019;15(6):556-559.

44. Yi J, et al. DNA damage-induced activation of CUL4B targets HUWE1 for proteasomal degradation. Nucleic Acids Res. 2015;43(9):4579-4590.

45. Zhong Q, Gao W, Du F, Wang X. Mule/ARF-BP1, a BH3-only E3 ubiquitin ligase, catalyzes the polyubiquitination of Mcl-1 and regulates apoptosis. Cell. 2005;121(7):1085-1095.

46. Sander B, Xu W, Eilers M, Popov N, Lorenz S. A conformational switch regulates the ubiquitin ligase HUWE1. Elife. 2017;6:e21036.

47. Broz P, von Moltke J, Jones JW, Vance RE, Monack DM. Differential requirement for Caspase-1 autoproteolysis in pathogen-induced cell death and cytokine processing. Cell Host Microbe. 2010;8(6):471-483.

48. Ball DP, et al. Caspase-1 interdomain linker cleavage is required for pyroptosis. Life Sci Alliance. 2020;3(3):e202000664.

49. Rodgers MA, et al. The linear ubiquitin assembly complex (LUBAC) is essential for NLRP3 inflammasome activation. J Exp Med. 2014;211(7):1333-1347.

50. Chen D, Kon N, Li M, Zhang W, Qin J, Gu W. ARF-BP1/Mule is a critical mediator of the ARF tumor suppressor. Cell. 2005;121(7):1071-1083.

51. King B, et al. The ubiquitin ligase Huwe1 regulates the maintenance and lymphoid commitment of hematopoietic stem cells. Nat Immunol. 2016;17(11):1312-1321.

52. Yang D, et al. HUWE1 controls the development of non-small cell lung cancer through down-regulation of $\mathrm{p} 53$. Theranostics. 2018;8(13):3517-3529.

53. Zhao X, et al. The N-Myc-DLL3 cascade is suppressed by the ubiquitin ligase Huwe1 to inhibit proliferation and promote neurogenesis in the developing brain. Dev Cell. 2009;17(2):210-221.

54. Kao SH, Wu HT, Wu KJ. Ubiquitination by HUWE1 in tumorigenesis and beyond. J Biomed Sci. 2018;25(1):67.

55. Cassidy KB, Bang S, Kurokawa M, Gerber SA. Direct regulation of Chk1 protein stability by E3 ubiquitin ligase HUWE1. FEBS J. 2020;287(10):1985-1999.

56. Mandemaker IK, et al. DNA damage-induced histone $\mathrm{H} 1$ ubiquitylation is mediated by HUWE1 and stimulates the RNF8-RNF168 pathway. Sci Rep. 2017;7(1):15353.

57. Moortgat $S$, et al. HUWE1 variants cause dominant X-linked intellectual disability(1): a clinical study of 21 patients. Eur J Hum Genet. 2018;26(1):64-74.

58. Friez MJ, et al. HUWE1 mutations in JubergMarsidi and Brooks syndromes: the results of an $\mathrm{X}$-chromosome exome sequencing study. $B M J$ Open. 2016;6(4):e009537.

59. Muthusamy B, et al. Exome sequencing reveals a novel splice site variant in HUWE1 gene in patients with suspected Say-Meyer syndrome. Eur JMed Genet. 2020;63(1):103635.

60. Schurz H, Salie M, Tromp G, Hoal EG, Kinnear CJ, Möller M. The X chromosome and sex-specific effects in infectious disease susceptibility. Hum Genomics. 2019;13(1):2.

61. Yang D, et al. Huwe1 Sustains normal ovarian epithelial cell transformation and tumor growth through the histone H1.3-H19 cascade. Cancer 
Res. 2017;77(18):4773-4784.

62. Guo C, et al. Cholesterol homeostatic regulator SCAP-SREBP2 integrates NLRP3 inflammasome activation and cholesterol biosynthetic signaling in macrophages. Immunity. 2018;49(5):842-856.e7.
63. Li F, et al. HECTD3 mediates TRAF3 polyubiquitination and type I interferon induction during bacterial infection. J Clin Invest. 2018;128(9):4148-4162.

64. Qi X, et al. Antagonistic regulation by the transcription factors C/EBP $\alpha$ and MITF spec- ifies basophil and mast cell fates. Immunity. 2013;39(1):97-110.

65. Zhao X, et al. The HECT-domain ubiquitin ligase Huwe1 controls neural differentiation and proliferation by destabilizing the $\mathrm{N}-\mathrm{Myc}$ oncoprotein. Nat Cell Biol. 2008;10(6):643-653. 